\title{
ANOMALIES IN THE APPLIED MAGNETIC FIELDS ON DIII-D AND THEIR IMPLICATIONS FOR THE UNDERSTANDING OF STABILITY EXPERIMENTS
}

\author{
by \\ J.L. LUXON, M.J. SCHAFFER, G.L. JACKSON, J.A. LEUER, \\ A. NAGY, J.T. SCOVILLE, and E.J. STRAIT
}




\section{DISCLAIMER}

This report was prepared as an account of work sponsored by an agency of the United States Government. Neither the United States Government nor any agency thereof, nor any of their employees, makes any warranty, express or implied, or assumes any legal liability or responsibility for the accuracy, completeness, or usefulness of any information, apparatus, product, or process disclosed, or represents that its use would not infringe privately owned rights. Reference herein to any specific commercial product, process, or service by trade name, trademark, manufacturer, or otherwise, does not necessarily constitute or imply its endorsement, recommendation, or favoring by the United States Government or any agency thereof. The views and opinions of authors expressed herein do not necessarily state or reflect those of the United States Government or any agency thereof. 


\title{
ANOMALIES IN THE APPLIED MAGNETIC FIELDS ON DIII-D AND THEIR IMPLICATIONS FOR THE UNDERSTANDING OF STABILITY EXPERIMENTS
}

\author{
by \\ J.L. LUXON, M.J. SCHAFFER, G.L. JACKSON, J.A. LEUER, \\ A. NAGY, J.T. SCOVILLE, and E.J. STRAIT
}

This is a preprint of a paper to be submitted for publication in Nuclear Fusion.

Work supported in part by the U.S. Department of Energy under

Contract Nos. DE-AC03-99-ER54463 and DE-AC02-76-CH03073 


\section{ABSTRACT}

Small non-axisymmetric magnetic fields are known to cause serious loss of stability in tokamaks leading to loss of confinement and abrupt termination of plasma current (disruptions). The best known examples are the locked mode and the resistive wall mode. Understanding of the underlying field anomalies (departures in the hardwarerelated fields from ideal toroidal and poloidal fields on a single axis) and the interaction of the plasma with them is crucial to tokamak development. Results of both locked mode experiments ${ }^{1}$ and resistive wall mode experiments ${ }^{2}$ done in DIII-D tokamak plasmas have been interpreted to indicate the presence of a significant anomalous field. New measurements of the magnetic field anomalies of the hardware systems have been made on DIII-D. The measured field anomalies due to the plasma shaping coils in DIII-D are smaller than previously reported ${ }^{3}$. Additional evaluations of systematic errors have been made. New measurements of the anomalous fields of the ohmic heating and toroidal coils have been added. Such detailed in situ measurements of the fields of a tokamak are unique. The anomalous fields from all of the coils are one third of the values indicated from the stability experiments 1,2 . These results indicate limitations in the understanding of the interaction of the plasma with the external field. They indicate that it may not be possible to deduce the anomalous fields in a tokamak from plasma experiments and that we may not have the basis needed to project the error field requirements of future tokamaks. 


\section{CONTENTS}

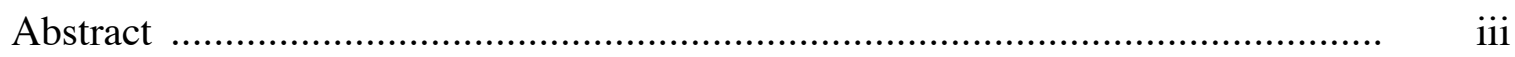

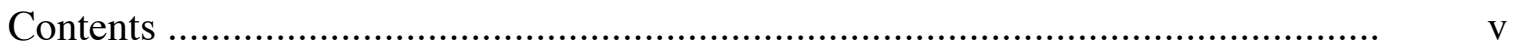

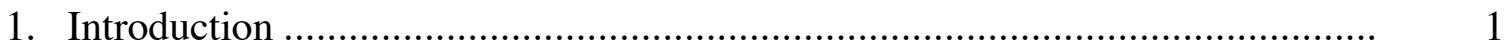

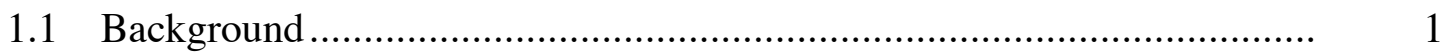

1.2 New Measurements on DIII-D............................................................. 2

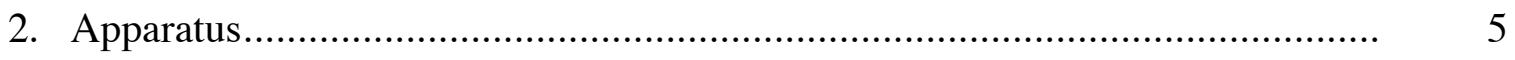

$2.1 \quad$ DIII-D Tokamak.........................................................................

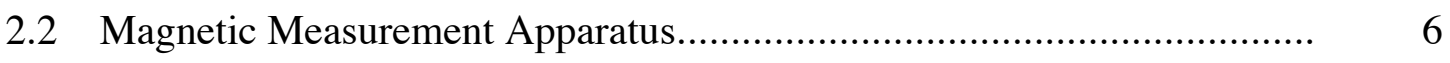

2.3 Methodology ……….................................................................. 9

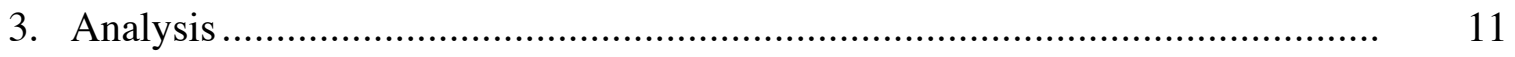

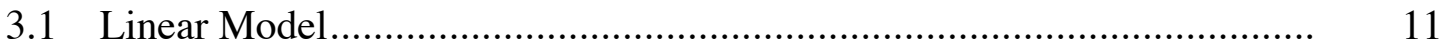

3.2 Perturbed Coil Model ..................................................................... 12

3.3 Combining Data Sets ...................................................................... 13

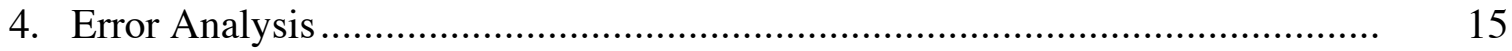

4.1 Statistical Uncertainty .................................................................... 15

$4.2 \quad$ Systematic Error ............................................................................... 16

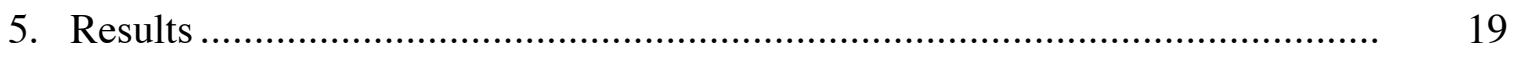

5.1 Plasma Shaping Coils ........................................................................ 19

5.2 Toroidal Field Coil ............................................................................ 21

5.3 Ohmic Heating Coil........................................................................... 24

5.4 Anomalies Expressed in the Toroidal Field Frame of Reference .............. 24

5.5 Harmonic Field Analysis ....................................................................... 28

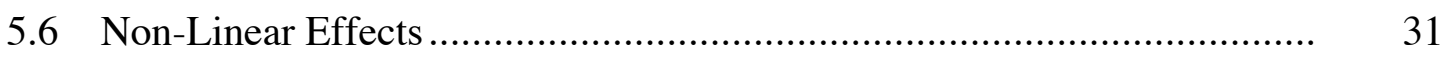

$5.7 \mathrm{n}=2$ and Higher Order Axial Fields....................................................... 32

6. Discussion and Summary ……................................................................. 35

6.1 Summary of Results ........................................................................ 35

6.2 Experimental Design .................................................................... 36

6.3 Relationship to DIII-D Plasma Experiments ............................................. 37

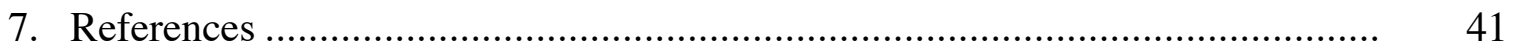

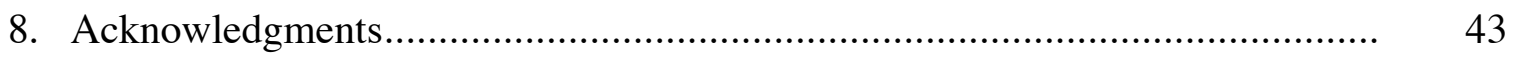




\section{INTRODUCTION AND OVERVIEW}

\subsection{Background}

Tokamaks and similar magnetic confinement devices depend on axisymmetric external magnetic fields for confinement, and small non-axisymmetric contributions to these fields can cause serious instabilities, loss of confinement, and lead to abrupt cessation of the plasma current. The best known examples of these instabilities include the lockedmode in which a low density rotating plasma slows and locks on to a field asymmetry and the unstable mode grows until confinement is $\operatorname{lost}^{3}$, and the resistive wall mode where the anomalous fields cause braking of the plasma rotation ${ }^{2}$. Non-axisymmetric error fields can also result in substantial errors in edge magnetic measurements and other diagnostics. In general, magnetic field components perpendicular to a plasma flux surface $B_{m n}$, where $m$ is the poloidal mode number and $\mathrm{n}$ is the toroidal mode number, with harmonic content $\mathrm{m}, \mathrm{n}=2,1$ are the most troublesome for plasma stability, although other nearby modes, especially 1,1 and 3,1 can contribute through viscous coupling4. Since very small field anomalies $\left(\delta \mathrm{B} / \mathrm{B}_{\mathrm{T}} \cong 10^{-4}\right.$, where $\mathrm{B}_{\mathrm{T}}$ is the toroidal field) can cause troublesome instabilities, small errors in the positioning of coils or owing to the coil leads or nearby magnetic objects can have serious consequences. As a result, careful attention must be paid to the positioning of coils in the design and construction of new devices, and additional "correction coils" may be needed to correct inadvertent field errors. This is of particular concern for reactor-size devices such as ITER 7 and FIRE ${ }^{8}$ where it appears necessary to limit field anomalies to even smaller values 9,7 . Recent measurements on JET use an extensive data set of plasma discharges coupled with a set of coils designed to produce only anomalous fields to deduce the contributions from the 2,1 fields and their parametric dependence on the plasma 6 .

Present understanding of magnetic confinement in the tokamak is that plasma equilibrium is dominated by the strong toroidal field. The fields of the other coil systems are assumed to be concentric with the toroidal field axis, and field contributions departing from this are treated as perturbations or anomalies and often described as error fields ${ }^{5}$.

DIII-D is a highly non-circular tokamak ${ }^{11}$ that has carried out a wide variety of seminal studies of tokamak stability ${ }^{12,13}$. The interpretation of these experiments depends critically on understanding the anomalies in the applied fields and how the plasma interacts with them. In this paper, we report measurements of the hardware-related anomalous (error) fields in DIII-D. These measurements were motivated by inconsistencies between 
the results of recent experiments and known error fields from the coil systems and inconsistencies between earlier magnetic measurements of the coil positions and mechanical measurements of the coils. Previous measurements in DIII-D of the dependence of locked-modes on field anomalies indicated that an unknown field $\mathrm{B}_{21}=7.1 \mathrm{G}$ at a toroidal field on axis $\mathrm{B}_{\mathrm{T} 0}=2.1 \mathrm{~T}$, was present beyond the fields that could be accounted for from previously measured coil positions and known to be present from toroidal coil buswork. This anomaly was related to the magnitude of the toroidal field ${ }^{1}$. Resistive wall mode experiments also were interpreted as indicating the presence of an anomalous field $\left(\mathrm{B}_{21} \cong 7 \mathrm{G}\right)^{2}$. Because these experiments indicate that the 2,1 mode is of greatest significance, the discussion in the remainder of the paper will emphasize this mode, but the results can be readily extended to others. Previous magnetic measurements of the coil positions and associated error fields ${ }^{3}$ were not consistent with mechanical measurements of the coil locations and did not address anomalies in the toroidal field or the ohmic heating coil field. Indeed, data from a number of diagnostics implied either the presence of an unknown field associated with the toroidal field coil, or a misalignment in the axes of the magnetic diagnostics (mounted on the plasma chamber) from the axis of the toroidal field of about $6 \mathrm{~mm}$. These characteristics have been stable over the intervening years.

DIII-D is the only major tokamak to have directly measured the non-axisymmetric field components from the coils and surrounding structure. COMPASS assembled their coil system using in situ measurements of the coil positions ${ }^{14}$. Other devices have inferred field anomalies from measurements of locked modes made over a wide range of parameters ${ }^{6}$. The results here will indicate that on DIII-D this procedure appears to have been misleading when used to determine the toroidal field related field anomalies.

\subsection{New Measurements on DIII-D}

The results reported here include measurements of the poloidal field coil related error fields, toroidal field coil error fields, non-linear effects, contributions from ferromagnetic sources, eddy currents, and static fields. The low order modes present on a flux surface cannot realistically be measured directly. Thus the technique used here and previously is to measure the $n=1$ perturbations on a circle near the plasma major radius, use these measurements to characterize the source of the anomaly for each coil, and then use this model to calculate the m,n harmonic components of the field averaged over a flux surface, e.g., $\mathrm{B}_{21}$ for the 2,1 mode. As was noted above, DIII-D stability experiments point to an anomalous field $\mathrm{B}_{21} \cong 7 \mathrm{G}$ at $\mathrm{B}_{\mathrm{T} 0}=2.1 \mathrm{~T}$. The corresponding $\mathrm{n}=1$ field on axis is typically $B_{1}=20 \mathrm{G}$. The experimental sensitivity to these fields is $\delta B_{21}= \pm 0.8 \mathrm{G}$ (in the locked mode experiments this corresponds to a change in the minimum plasma density for locked mode onset of $2.5 \times 10^{13} \mathrm{~cm}^{-3}$ ). Thus identification of coil-related sources requires measurements of $B_{1}$ to an accuracy of better than $\pm 20 \mathrm{G}$, and measurements to an 
ANOMALIES IN THE APPLIED MAGNETIC FIELDS ON

DIII-D AND THEIR IMPLICATIONS FOR THE

UNDERSTANDING OF STABILITY EXPERIMENTS

J.L. Luxon, et al.

accuracy of $\pm 1 \mathrm{G}$ would be useful. In the case of a simple circular plasma shaping coil shifted from the axis of the toroidal field by an unknown amount, the location of the coil is determined from the three $n=1$ field components. The resultant shift from an axial configuration is then used to calculate the higher order components, e.g., $\mathrm{B}_{21}$. Typically, $\mathrm{B}_{21} \tilde{<} 0.5 \mathrm{G} / \mathrm{cm}$ for a mis-positioned plasma shaping coil at $2 \mathrm{kA}$, and this can be determined to an accuracy of $10 \%$.

DIII-D has three major coil systems, the toroidal field coil, the ohmic heating coil, and the plasma shaping coils (Fig. 1).

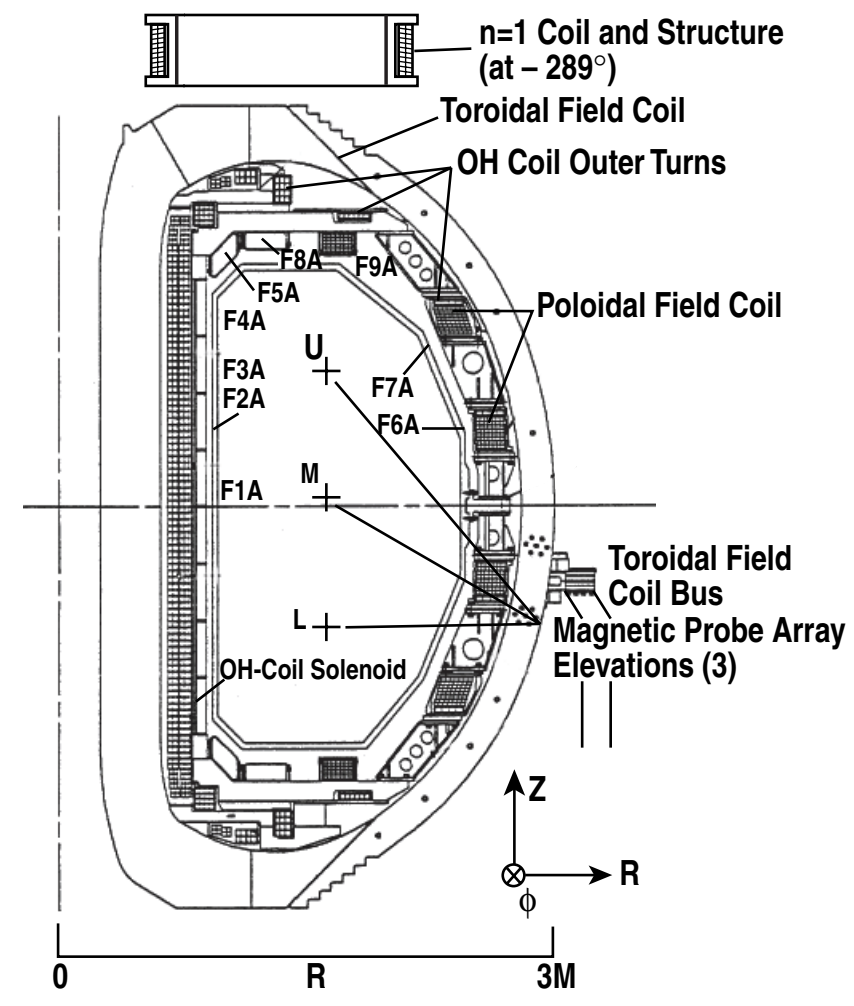

Fig. 1. The cross-section of DIII-D showing the coil positions and the elevations of the measurement apparatus. The shaping coils in the lower half of the tokamak are labeled F1B to F9B in mirror image of the upper half.

The toroidal coil produces the highest fields and carries the highest currents. The anomalies in the toroidal field are most difficult to measure because of the high background field. As will be seen, our ability to measure $n=1$ components of the toroidal field was limited to about $6 \mathrm{G}$. The plasma shaping coils consist of 18 poloidal coils used to position and shape the plasma. They are nominally centered on the toroidal coil to within $1 \mathrm{~cm}$ in the horizontal plane and $0.02^{\circ}$ of tilt. Typical fields at $\mathrm{R}_{0}$ are $\mathrm{B}_{\mathrm{P}}<1 \mathrm{kG}$. The expected $\mathrm{n}=1$ fields are $\mathrm{B}_{1} / \mathrm{B}_{\mathrm{P}} \cong 10^{-5} / \mathrm{mm}$ of displacement. The aggregate measured 2,1 field of all the shaping coils in their installed positions as reported herein is of order $\mathrm{B}_{21}=1 \mathrm{G}$ for a $1 \mathrm{MA}$ discharge and can be determined to an accuracy of $10 \%$. The DIII-D ohmic heating 
coil consists of a set of 122 toroidal turns surrounding the plasma region and designed to provide flux change in the central bore to drive the plasma current while producing little $\mathrm{n}=0$ field in the plasma region. Nevertheless, if the component coils are out of position with respect to the toroidal axis, they produce anomalous fields. Measurements of these anomalies are made difficult by the noise related to the large amount of flux in the system, and analysis is complicated by the spatial distribution of the component turns. At full current (137 kA), $\mathrm{B}_{1} \cong 3-5$ Gauss $\pm 10 \%$. On the other hand, for many discharges used in stability studies, the currents in this coil at the time of interest are small. From this introduction, it can be seen that the toroidal field coil anomalies will have a strong role in determining our understanding of the magnetic field anomalies.

Careful attention was paid to assess the role of ferromagnetic materials. Indeed, during preparation for these measurements, a large previously unrecognized ferromagnetic structure was identified, characterized, and permanently removed from the vicinity of the tokamak. The measurements reported here were made in the interval between two run campaigns and no field-related changes in the operation of the tokamak were noted.

In Section 2, we will discuss the apparatus, including the DIII-D tokamak and the probe array placed in the plasma chamber for the measurements. Section 3 describes the methods used to analyze the data, while Section 4 covers the uncertainty in the results including propagation and evaluation of systematic contributions. Section 5 presents the results for the $n=1$ fields characterizing DIII-D and their propagation to higher order fields. Finally, Section 6 gives a summary and discussion of the conundrum presented by the fact that the results here increase the discrepancy between the field anomalies indicated by stability experiments and the measured values. 


\section{APPARATUS}

\subsection{DIII-D Tokamak}

DIII-D is a non-circular cross-section tokamak ${ }^{11}$ with major radius $\mathrm{R}_{0}=1.66 \mathrm{~m}$, minor half width $\mathrm{a}=0.66 \mathrm{~m}$, and maximum plasma elongation of $\sim 2.5$ (Fig. 1). The toroidal coil consists of 144 turns in 24 bundles with a maximum current of $126 \mathrm{kA}$ producing a field at $\mathrm{R}_{0}$ of $\mathrm{B}_{\mathrm{T} 0}=2.2 \mathrm{~T}$. The two current feeds consist of single conductor pairs separated on average by about $0.2 \mathrm{~m}$ (a linear dipole) and have a small but non-negligible error field. The contributions of this buswork to the magnetic field have been modeled based on engineering drawings along with recent visual confirmation of their geometry. The $\mathrm{n}=1$ field at $\mathrm{R}_{0}$ is $\mathrm{B}_{1} \cong 5 \mathrm{G}$ and $\mathrm{B}_{21}=1.7 \mathrm{G}$ at $250^{\circ}$. The leads of the poloidal field coils are connected in a quadrapole configuration (two leads in and two leads out) and produce very little contribution to the anomalous fields ${ }^{11,3}$.

Right-handed cylindrical coordinates are used throughout this report. The DIII-D facility identifies component locations with respect to a geographic (left-handed) coordinate system. To rationalize the two coordinate systems, $0^{\circ}$ is taken as that used by the DIII-D facility, and all DIII-D facility locations are described with negative sign to properly locate them in the right-handed coordinate system.

The so-called $n=1$ coil, was placed on top of the machine (1989) to provide a source of non-axisymmetric field. It was approximately $1.8 \mathrm{~m}$ in diameter and located at $\mathrm{R}=1.59 \mathrm{~m}, \mathrm{z}=2.86 \mathrm{~m}$, and at $-289^{\circ}$ toroidally. During preparation for these studies, it was found that this coil was supported by a ferromagnetic structure consisting roughly of an open cylinder $1.8 \mathrm{~m}$ in diameter and $0.30 \mathrm{~m}$ high. This structure was large enough to cause substantial shifts to the measurements reported here and previously ${ }^{3}$. The contribution of this ferromagnetic structure to plasma operations is determined by its polarization in the total local poloidal field and cannot be determined from individual coil measurements. The ferromagnetic structure is estimated to have caused negligible perturbations to plasma experiments owing to the large poloidal fields present in the plasma chamber. It was removed as part of a systematic study in the early part of these measurements. The absence of the $n=1$ structure caused no observed change in subsequent plasma operation.

In addition, a number of relatively small ferromagnetic structures are located in the vicinity of the tokamak. Of most concern are a number (15) of $0.5 \mathrm{ft}^{3}\left(14000 \mathrm{~cm}^{3}\right)$ shielded enclosures for pyrometers and infrared camera located just outside the poloidal 
shaping coils between F9A and F9B and as near as $20 \mathrm{~cm}$ from the outer surface of a poloidal coil. The largest object is a shielded room $18 \mathrm{~m}^{3}$ in volume at $\mathrm{R}=6.4 \mathrm{~m}$ and $\mathrm{z}=-4.1 \mathrm{~m}$ at $135^{\circ}$. There is also significant iron in the support stands and bending magnets of the neutral beamlines located at $30^{\circ}, 150^{\circ}, 210^{\circ}$, and $330^{\circ}$, but this has $\mathrm{n}=2,4$, and 6 symmetry with little $n=1$.

To provide an additional source of non-axisymmetric magnetic fields for error field optimization during plasma experiments, a set of six picture frame coils is installed in a band at the midplane outside the toroidal field coil at $\mathrm{R}=3.2 \mathrm{~m}$. The coils are approximately $60^{\circ}$ in toroidal extent $(3.2 \mathrm{~m})$ and have a height of $1.6 \mathrm{~m}$. They are connected in anti series and are capable of producing a roughly radial field perturbation of up to $15 \mathrm{G}$ on the midplane at $\mathrm{R}_{0}$. Generally, the currents in these coils can be adjusted to produce an $n=1$ spectrum peaked at low $m$ at arbitrary angle with unavoidable lesser sidebands. The field from these coils is used to optimize the characteristics of the plasma based on predetermined inputs and empirical adjustment. They also provide an excellent reference field to check the operation of the probe array used for these measurements.

The DIII-D plasma chamber is a continuous, toroidally symmetric, multi-layered shell made of Inconel. It is supported from four points on the outer midplane by the outer poloidal coil support structure. Construction measurements indicated that the vessel is toroidally symmetric within an rms deviation of $\pm 1 \mathrm{~mm}$. In addition the inner bore is offset from the outer wall by $\sim 1 \mathrm{~mm}$ at $-75^{\circ}$. Magnetic measurements from vessel-mounted probes along with measurements associated with a number of diagnostic systems made over many years, indicate that it is a stable structure. One such example is the measurement of the relative position of the vacuum vessel and the toroidal field coil discussed below, which has been stable for over at least ten years and likely since construction.

\subsection{Magnetic Measurement Apparatus}

The majority of the data reported here was taken using an in-vessel array of probes measuring the radial, toroidal, and vertical components of the field, $\mathrm{B}_{\mathrm{R}}, \mathrm{B}_{\phi}$, and $\mathrm{B}_{\mathrm{Z}}$, respectively, at eight equally spaced points on the circumference of a circle at $\mathrm{R}_{\mathrm{m}}=1.64 \mathrm{~m}$ (Fig. 2). The individual probes are rectangular spirals mounted on a composite fiber board made using printed circuit board technology 16 . This provides a high degree of uniformity and a rigid geometry. The $\mathrm{B}_{\mathrm{R}}$ and $\mathrm{B}_{\phi}$ probes had an effective area of $0.796 \mathrm{~m}^{2}$ and the $\mathrm{B}_{\mathrm{Z}}$ probes $1.60 \mathrm{~m}^{2}$. The rms deviations in the data indicate that the probes are equal to within at least $3.5 \times 10^{-4}$ (see Table 1 below). The only measurement for which this is of any consequence is the determination of the location of the toroidal field center. 
The probe support structure was made of non-magnetic materials. The support ring was made of fiber reinforced resin I-beam sections fastened together with rigid fiberglass composite corner plates using tight fitting sleeves for alignment. The structure had to be demountable in order to pass it through the entry port into the plasma chamber. One $B_{R}$ and one $\mathrm{B}_{\phi}$ probe were

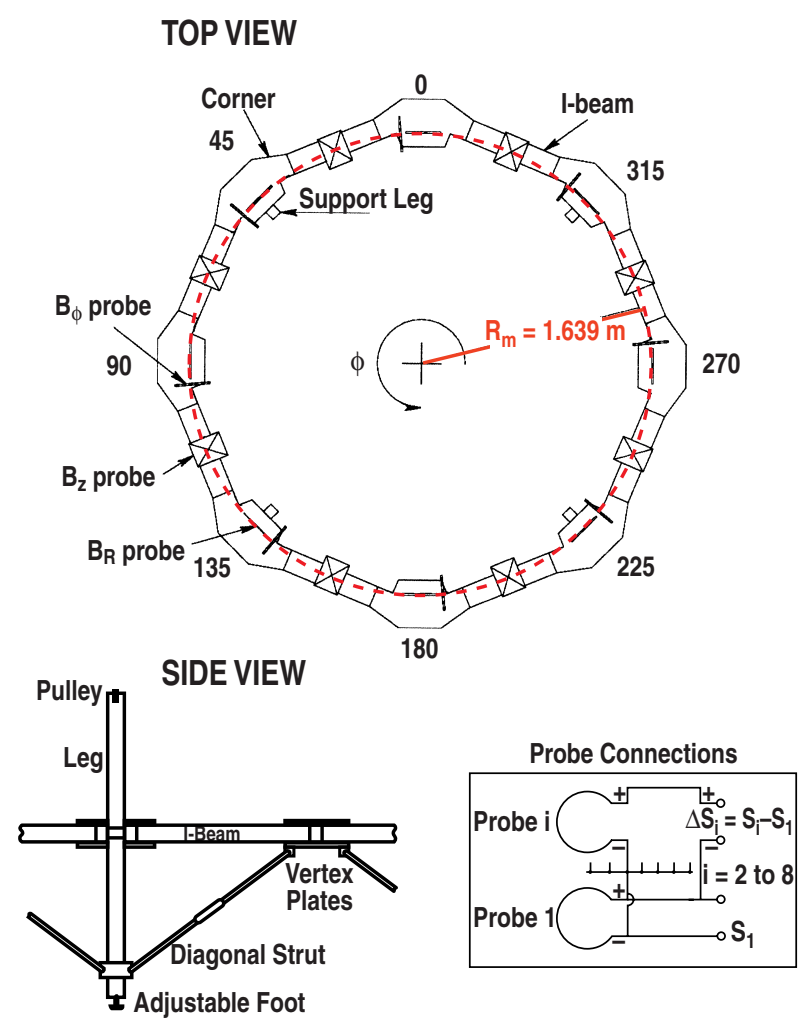

Fig. 2. A schematic representation of the measurement apparatus. Inset is the probe connection scheme used to reject the $\mathrm{n}=0$ component.

Table 1: Random error contributions in the magnetic probe data. Evaluated from the rms variations in the 8 point data sets.

\begin{tabular}{|l|c|c|c|c|}
\hline \hline SOURCE $>$ & \multicolumn{1}{|c|}{$\mathrm{B}_{\mathrm{R}}$} & \multicolumn{1}{c|}{$\mathrm{B}_{\mathrm{T}}$} & $\mathrm{B}_{\mathrm{Z}}$ & (const.) \\
\hline & $\left(10^{-3}\right)$ & $\left(10^{-3}\right)$ & $\left(10^{-3}\right)$ & $\left(10^{-3} \mathrm{G}\right)$ \\
MEASURED & 0.35 & 1 & 1 & 20 \\
$\sigma_{\mathrm{BR}}$ & 2 & 0.35 & 0.5 & 20 \\
$\sigma_{\mathrm{BT}}$ & 1 & 0.6 & 0.35 & 20 \\
$\sigma_{\mathrm{BZ}}$ & \multicolumn{4}{|l}{} \\
e.g., $\sigma_{\mathrm{BR}}\left(10^{-3} \mathrm{G}\right)=.35 \mathrm{~B}_{\mathrm{R}}+1.0 \mathrm{~B}_{\mathrm{T}}+1.0 \mathrm{~B}_{\mathrm{Z}}+20$ \\
\hline \hline
\end{tabular}


mounted on each corner plate so that the probes could be aligned outside the vessel, the corner plate disassembled, and then reassembled inside the plasma chamber while maintaining the probe alignment. The $\mathrm{B}_{\mathrm{Z}}$ probes were mounted at the centers of the beams. The structure was supported on four adjustable legs to allow it to be set at different elevations and diagonal struts gave it rigidity.

The entire apparatus was first assembled outside the plasma chamber and aligned. The frame was found to be quite rigid and reproducible in the radial direction $( \pm 0.5 \mathrm{~mm})$, but somewhat more flexible in the vertical direction $( \pm 2 \mathrm{~mm})$, and care had to be taken to realign it after each change in position. The probes were installed on the frame with a precision of $\pm 0.5 \mathrm{~mm}$ in $\mathrm{R}, \pm 0.2 \mathrm{~mm}$ in $\mathrm{z}$ and $\pm 0.04^{\circ}$ in $\phi$. The individual probe surfaces were then aligned to vertical with an accuracy of $\pm 0.025^{\circ}$, and to radial to \pm 0.04 .

The apparatus was then assembled inside the plasma chamber. A set of eight radial scales was fastened to the center wall of the chamber near the floor. The apparatus was centered in the horizontal plane to a reproducibility of $\pm 1.0 \mathrm{~mm}$ using plumb bobs in conjunction with these scales. The vertical position was set with an accuracy of $\pm 1 \mathrm{~mm}$ relative to the vessel floor. Similarly, the azimuth of the entire apparatus was set with an accuracy of $0.5^{\circ}$ using known fiducials on the wall. Following this, the apparatus was aligned to be horizontal to $\pm 0.03^{\circ}$. It was found that the probes did not maintain their positions to the expected accuracy and so they were realigned with respect to horizontal and vertical to $\pm 0.025^{\circ}$ every time the apparatus was moved. There was no mechanism to realign the probes in the $\mathrm{R}$ and $\phi$ directions once the apparatus was installed in the plasma chamber. This is most significant for the measurements of $B_{R}$ in the presence of the strong toroidal field of the toroidal field coil. The best measure of the random and systematic error in the apparatus alignment is found in the rms deviation in the data from the $n=1$ and $n=2$ components as discussed in Section 4 below.

Additional magnetic measurements are available from both toroidal and poloidal arrays of permanently installed saddle loops fastened to the wall of the plasma chamber. These arrays are valuable in determining the location of the chamber with respect to the toroidal field and for evaluating non-linear effects with both the toroidal and poloidal coils energized. The poloidal saddle loop array consists of two sets of 11 quasi-rectangular loops, each in a poloidal band on the chamber wall. Each loop is approximately $30^{\circ}$ in arc toroidally and $0.6 \mathrm{~m}$ in poloidal extent. One array is located at $-67^{\circ}$ toroidally and the other at $-157^{\circ}$. An analysis of the rms variations in the data indicates that these saddle loops are positioned to an accuracy of $\pm 1 \mathrm{~mm}$ in the radial direction. The toroidal array consists of 18 loops on the plasma-facing side of the outer vessel wall. They are arranged with six loops around the circumference of the vessel, each approximately $60^{\circ}$ in arc $(2.5 \mathrm{~m})$ wide and three high symmetrically about the midplane. The loops have a height in the poloidal direction of $0.8 \mathrm{~m}$. The rms variations in the data from these loops are 
ANOMALIES IN THE APPLIED MAGNETIC FIELDS ON

DIII-D AND THEIR IMPLICATIONS FOR THE

UNDERSTANDING OF STABILITY EXPERIMENTS

J.L. Luxon, et al.

equivalent to a placement error of $\pm 3 \mathrm{~mm}$ in the conductors. Thus they have a poorer placement accuracy than the poloidal array, but the ability to average over the entire set results in being able to achieve similar accuracy for measurements of the toroidal field. Although the locations of these loops are too poorly understood to use them for a priori measurements of the poloidal coil positions, once they have been referenced to a better understood set of measurements, such as those presented here (to remove systematic positioning errors), they can be used as a baseline for changes in the coil-related fields or the sources producing them.

\subsection{Methodology}

In order to improve the accuracy of the measurements of the upper and lower plasma shaping coils and to aid in identifying systematic errors, the apparatus was positioned at three different elevations $\mathrm{z}=-0.75 \mathrm{~m}, \mathrm{z}=+0.044 \mathrm{~m}$, and $\mathrm{z}=+0.75 \mathrm{~m}$, denoted $\left(\mathrm{L}, \mathrm{M}_{0}\right.$, and $\mathrm{U}$ respectively). In addition, at the midplane elevation, the apparatus was rotated toroidally through an angle of $65^{\circ}$ (denoted $\mathrm{M}_{65}$ ), which was large enough to identify systematic errors in the apparatus when measurements were compared to measurements at $\mathrm{M}_{0}$ and was near an odd half-multiple of the spacing between probes $\left(45^{\circ}\right)$. Thus, the $M_{0}$ and $\mathrm{M}_{65}$ eight-point midplane data sets can be combined into a single 16 point data set to allow $n$ values as large as $n=7$ to be identified. The data sets were taken in the order $L, M_{0}, U$, and $\mathrm{M}_{65}$ at the rate of one set/day.

Data collection was optimized for detecting the relatively small values of each component of $\mathbf{B}$ for $\mathrm{n} \geq 1$ ( $\leq 2 \mathrm{G}$ for the poloidal coils) compared to the background or $\mathrm{n}=0$ level ( $\leq 200 \mathrm{G}$ for the poloidal coils). This was achieved by using the first probe for each field component as the reference and wiring each of the remaining seven probes to electrically subtract the reference signal before integrating and recording the values (see inset Fig. 2). The resulting signals from all 24 probes were then integrated and digitized. Standard components from the DIII-D data acquisition system were used for this, and the data was recorded in the DIII-D database using the standard time base. The coil current was turned on and regulated to be constant. Sufficient time $(1.5 \mathrm{~s}$ for poloidal field coils and $5 \mathrm{~s}$ for the toroidal field coil) was allowed for any time dependent terms $(<5 \%)$ to decay. The value of a particular probe signal for a given coil along with the coil current were determined by averaging over an interval of $\sim 1 \mathrm{sec}$ near the end of the coil pulse after the signal had reached steady state. The value of the baseline at times drifted during the pulse owing to drift in the integrator, and this was removed to lowest order by automatically subtracting the baseline calculated using a linear ramp model. The accuracy of the acquired signals was better than $0.1 \%+0.02 \mathrm{G}$. A typical data set is shown in Fig. 3 .

The data set was assembled by individually pulsing each coil. The field shaping coils were normally operated at about $2000 \mathrm{~A}$ and occasionally operated at $4000 \mathrm{~A}$. The ohmic 


\section{J.L. Luxon, et al.}

ANOMALIES IN THE APPLIED MAGNETIC FIELDS

ON DIII-D AND THEIR IMPLICATIONS FOR THE

heating coil was normally operated at $20 \mathrm{kA}$, but occasionally at $100 \mathrm{kA}$. The toroidal field coil was operated at $20 \mathrm{kA}$, although on several occasions it was operated at $120 \mathrm{kA}(2.1 \mathrm{~T})$. For typical discharges in DIII-D, currents in the outer field shaping coils are as large as $10 \mathrm{kA}$ (at a plasma current of $2.5 \mathrm{MA}$ ), while the inner poloidal field coil currents are normally no more than $5 \mathrm{kA}$.
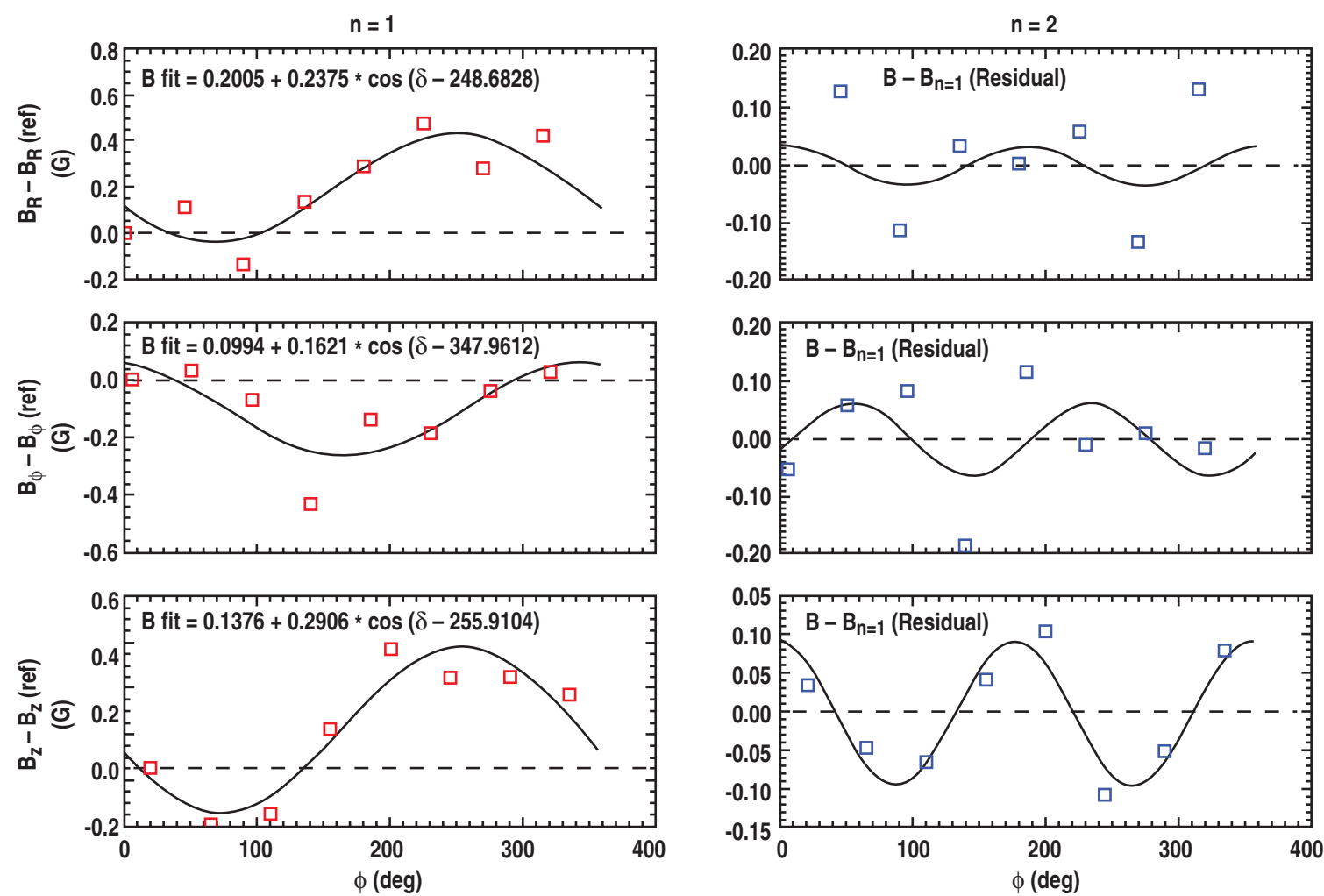

Fig. 3. The data set for F3B at $M_{0}$ illustrating individual fits of the $n=1$ component to the measured values and the $\mathrm{n}=2$ component to the residual. The data was averaged over the interval $5000 \mathrm{~ms}$ to $6400 \mathrm{~ms}$. The squares represent the data points and the solid lines are the fit. 


\section{ANALYSIS}

Using a Fourier expansion, each field component can be fit to a function of the form $\mathrm{B}_{\mathrm{x}}(\phi)=\Sigma_{\mathrm{n}} \mathrm{B}_{\mathrm{nx}} \cos \left(\mathrm{n} \phi-\phi_{\mathrm{n}}\right)$, with $0<\mathrm{n}<7$ and $\mathrm{x}$ denoting $\mathrm{R}, \phi$, or $\mathrm{z}$. It is expected from Fourier analysis that values of $n$ up to $n=3$ can be meaningfully determined. The $n=0$ component for each coordinate is recorded to first order by the reference probe. The $n=1$ data is fit to models for a tilted and shifted coil using two different techniques, the linear model and the perturbed coil model. The use of the two models very much complemented each other, and in the final analysis they produced identical answers to within a small fraction of a standard deviation. The $n=2$ data can be used to determine the ellipticity and bowing of the coil, while $n=3$ measures the triangularity. The data set for each coil at each apparatus location has six degrees of freedom each for $n=1,2$, and 3 .

\subsection{Linear Model}

The linear model used a linear expansion for small shifts and tilts of a single-turn at the center of each coil, and was similar to that developed previously ${ }^{3}$ with the addition that all three components of the field were analyzed to make full use of the data. The shift of a coil is defined by $\boldsymbol{d}=d e^{i \phi_{d}}$ and tilt by $\boldsymbol{\alpha}=\alpha e^{i \phi_{\alpha}}$, where $\boldsymbol{d}$ is the shift of the coil axis in the horizontal plane of the apparatus toward toroidal angle $\phi_{\mathrm{d}}$ and $\boldsymbol{\alpha}$ is the upward tilt of the coil horizontal plane from horizontal at toroidal angle $\phi_{\alpha}$ (Fig. 4).

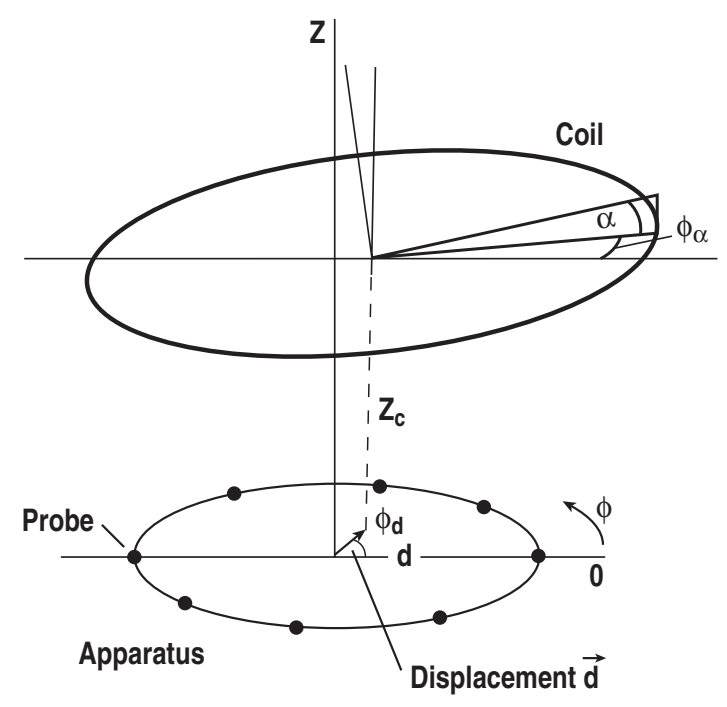

Fig. 4. Geometry of the shift and tilt of a poloidal coil in the coordinates of the measuring apparatus. 
For small poloidal field coil perturbations, the linearized equations for the three field components of the $n=1$ field are given by

$$
\left[\begin{array}{l}
\mathrm{B}_{1 R} \\
\mathrm{~B}_{1 \phi} \\
\mathrm{B}_{1 z}
\end{array}\right]=\left[\begin{array}{lc}
-d \frac{\partial}{\partial \mathrm{R}} \cos \left(\phi-\phi_{d}\right)-\alpha\left(z_{c} \frac{\partial}{\partial \mathrm{R}}+\mathrm{R}_{m} \frac{\partial}{\partial z}\right) \cos \left(\phi-\phi_{\alpha}\right) 0-\alpha \cos \left(\phi-\phi_{\alpha}\right) \\
\frac{d}{\mathrm{R}_{m}} \sin \left(\phi-\phi_{d}\right)+\frac{\alpha z_{c}}{\mathrm{R}_{m}} \sin \left(\phi-\phi_{\alpha}\right) & 0+\alpha \sin \left(\phi-\phi_{\alpha}\right) \\
\alpha \cos \left(\phi-\phi_{\alpha}\right) & 0-d \frac{\partial}{\partial \mathrm{R}} \cos \left(\phi-\phi_{d}\right)-\alpha\left(z_{c} \frac{\partial}{\partial \mathrm{R}}+\mathrm{R}_{m} \frac{\partial}{\partial z}\right) \cos \left(\phi-\phi_{\alpha}\right)
\end{array}\right]\left[\begin{array}{l}
\mathrm{B}_{R} \\
\mathrm{~B}_{\phi} \\
\mathrm{B}_{\mathrm{z}}
\end{array}\right],
$$

where $z_{c}$ is the distance from the center of the coil to the plane of the apparatus. For toroidal field coil perturbations:

$$
\left[\begin{array}{c}
\mathrm{B}_{1 R} \\
\mathrm{~B}_{1 \phi} \\
\mathrm{B}_{1 \mathrm{z}}
\end{array}\right]=\left[\begin{array}{ll}
0-\frac{d}{\mathrm{R}_{m}} \sin \left(\phi-\phi_{d}\right)-\frac{\alpha z_{c}}{\mathrm{R}_{m}} \sin \left(\phi-\phi_{\alpha}\right) & 0 \\
0 & +\frac{d}{\mathrm{R}_{m}} \cos \left(\phi-\phi_{d}\right)+\frac{\alpha z_{c}}{\mathrm{R}_{m}} \cos \left(\phi-\phi_{\alpha}\right)
\end{array}\right]\left[\begin{array}{l}
\mathrm{B}_{R} \\
0-\alpha \sin \left(\phi-\phi_{\alpha}\right)
\end{array}\right]\left[\begin{array}{c}
B_{1 H} \cos \left(\phi-\phi_{H}\right) \\
\mathrm{B}_{\phi} \\
\mathrm{B}_{\mathrm{z}}
\end{array}\right]+\left[\begin{array}{c}
-B_{1 H} \sin \left(\phi-\phi_{H}\right) \\
B_{1 V} \cos \left(\phi-\phi_{V}\right)
\end{array}\right]
$$

where we have included the possibility of an $n=1$ field $B_{1 H}$ in the horizontal plane at angle $\phi_{\mathrm{H}}$ and an $\mathrm{n}=1$ vertical field $\mathrm{B}_{1 \mathrm{~V}}$ at angle $\phi_{\mathrm{V}}$. The simplest form of $\mathrm{B}_{1 \mathrm{H}}$ is a uniform transverse field, which results in an $n=1$ field in $R$ and $\phi$. This field has the effect of separating the apparent centers of the toroidal field as measured by $B_{\phi}$ and $B_{R}$. Note that these equations contain only $n=1$ dependence on $\phi$. Also note from the equations for the toroidal field, that the $\mathrm{z}$ component determines the sum of the tilt of the axis and $\mathrm{B}_{1 \mathrm{~V}}$.

For the plasma shaping coils, these equations are over-specified for determining $\boldsymbol{d}$ and $\boldsymbol{\alpha}$ there are 4 unknowns and 6 degrees of freedom), and a least-square minimization was used to simultaneously fit the four unknowns to the data set. For the toroidal coil, $\boldsymbol{d}$ and $\mathbf{B}_{1 \mathrm{H}}$ are determined from $\mathrm{B}_{1 \phi}$ and $\mathrm{B}_{1 \mathrm{R}}$, and $\boldsymbol{\alpha}$ is determined from $\mathrm{B}_{1 \mathrm{Z}}$. This model was programmed using IDL and efficiently processed the large sets of data on a large computer.

\subsection{Perturbed-Coil Model}

The perturbed-coil model calculates displacements and tilts of one-turn approximations for the coils to model the expected fields without linearizing assumptions. The poloidal coils are described as circular turns in the frame of reference of the probe set and the fields are calculated using standard elliptical integral techniques. Again, the equations are over-specified and the best shift and tilt of the coil to fit the experimental data is calculated using a least square minimization of the difference of the model calculations at each probe from the experimental measurements. The toroidal coil is modeled as a single 
ANOMALIES IN THE APPLIED MAGNETIC FIELDS ON

DIII-D AND THEIR IMPLICATIONS FOR THE

UNDERSTANDING OF STABILITY EXPERIMENTS

J.L. Luxon, et al.

axial filament. These calculations are carried out in an Excel spread sheet. This technique had the advantage that it was much easier to add features to the model such as the existence of a ferromagnetic dipole, and to do systematic studies and error analysis.

A model for a ferromagnetic dipole was added to the perturbed-coil model. The dipole is assumed to be in the form of a sphere of material with infinite permeability of radius $a$ centered at a point. The dipole moment is calculated as a function of $a$ and the magnitude and direction of the exciting field from the coil in use. The dipole moment is then used to calculate the field contributions at each of the probes and these are added to the model. A sufficiently large individual dipole can be fit to the data in the same manner as the position of the coil was fit. In practice, this was found to be difficult to do because of the small size of the dipoles, the highly non-linear nature of the dipole, and the fact that its main contributions to the field were dominant at only one or two probe positions $(n \cong 8)$. Nevertheless, the model was very useful in estimating the impact of ferromagnetic structures on measured coil positions.

\subsection{Combining Data Sets}

Once the positions of the plasma shaping coils are determined for each of the four data sets, these data sets are combined into one overall assessment of the relative positions of the coils to a common axis. In order to do this, the plasma shaping coils are taken as determining a common three dimensional reference frame. The center of the probe array for the $\mathbf{M}_{0}$ data set is taken as the origin and the normal to plane at this point is taken as defining vertical. The other data sets are then transformed to this single axis. This is done using a weighted least square minimization of the differences between location and tilt of each of the coils in the other data sets to the reference data set. In this manner, we were able to make small corrections to the recorded positions of the apparatus (of the order of $1 \pm 0.35 \mathrm{~mm}$ and $.04 \pm 0.015^{\circ}$ ) that improved upon our ability to position the apparatus manually. Once this is done there are four measurements for each coil position in a common reference frame. These are then used to determine a best location for each coil. These locations were also used in combining the four data sets for the toroidal field coil axis.

The data from the poloidal saddle loop array was fit using a simple geometric model for the shift of the loops in a toroidal field. This gives the shift of the axis of the loop array and thus the plasma chamber with respect to the axis of the toroidal field. The toroidal array is conceptually simpler to fit since each loop lies on a surface of revolution, and the variation is expected to be sinusoidal $(n=1)$. A least-square minimization was used to calculate the $n=1$ component. 


\section{UNCERTAINTY ASSESSMENT}

\subsection{Statistical Uncertainty}

The uncertainty in the determinations of $\boldsymbol{d}$ and $\boldsymbol{\alpha}$ are due to a combination of the accuracy of the probes $\left(3.5 \times 10^{-4}\right)$, the positioning of the probes $(0.5 \mathrm{~mm} / 1640 \mathrm{~mm} \mathrm{in} \mathrm{R})$, and the angular alignment of the probes $\left(5 \times 10^{-4}\right)$. The use of differential techniques in the collection of the eight point data sets made contributions to the error from the amplification and integration of the signals insignificant. This leads us to an expected a priori error in the probe measurements of $\sigma / \mathrm{B} \cong 7 \times 10^{-4}$. The achieved error in the measurements for a given coil can be estimated from the rms variation of the individual data points from the fitted functions. The rms variation in the probe signals after the $n=1$ and $n=2$ components are accounted for, is typically $\sigma / \mathrm{B} \cong 1 \times 10^{-3}$. The contributions to the error from the various components of the fields, made from an a posteriori assessment of the data are summarized in Table 1.

The uncertainty in the fitted parameters is evaluated by determining the point where the minimum in $\chi^{2}$ increases by 1 . The uncertainty was smallest when the apparatus was nearest the coil (e.g., F6A or F1B measured from $\mathrm{M}_{0}$ ). For measurements with the apparatus farther away, the uncertainty increased significantly. The uncertainties range from $0.3 \mathrm{~mm}$ and $0.01^{\circ}$ for F6A and F6B to $0.8 \mathrm{~mm}$ and $0.044^{\circ}$ for F9A and F9B. Here uncertainties in the vector quantities are quoted as one dimension which characterizes a circle around the point in the two dimensional plane. For most of the measurements, the variables $\boldsymbol{d}$ and $\boldsymbol{\alpha}$ were essentially independent. However, for the measurements made with the apparatus positioned furthest from the coil (e.g., F5A, F8A, or F9A from the $\mathrm{L}$ position), the codependence was significant. This, coupled with the already large uncertainty, resulted in such measurements being of little value. Once the apparatus was set in a given position, the reproducibility of repeated measurements was better than \pm 0.1 $\mathrm{mm}$ and $0.004^{\circ}$.

The two analysis techniques realized very similar results. The standard deviation of the difference was less than $\pm 0.25 \mathrm{~mm}$ for $\boldsymbol{d}$ and $\pm 0.02^{\circ}$ for $\boldsymbol{\alpha}$. This is insignificant on the scale of the error associated with each quantity.

The four data sets were transformed to a common axis and the best-estimate position for each coil was calculated using a weighted average. The uncertainties were calculated including a small contribution due to the transformation to the $\mathrm{M}_{0}$ axis. A measure of the 
ANOMALIES IN THE APPLIED MAGNETIC FIELDS ON

DIII-D AND THEIR IMPLICATIONS FOR THE

UNDERSTANDING OF STABILITY EXPERIMENTS

J.L. Luxon, et al.

F7A, $\boldsymbol{d}$ changed by $2.76 \mathrm{~mm}$ at $251^{\circ}$ and $\boldsymbol{\alpha}$ by $0.06^{\circ}$ at $230^{\circ}$. Note that the measured changes in location are all consistent with the location of the $n=1$ structure at $-289^{\circ}$, from which we would expect the phase of the perturbation to be $360^{\circ}-289^{\circ}+180^{\circ}=251^{\circ}$.

The DIII-D machine hall is covered by a removable roof structure that contains ferromagnetic material. It is located at $\mathrm{z} \cong 5 \mathrm{~m}$. Comparing measurements made with the roof both on and off, this structure was found to make no significant contribution to the error fields.

A systematic study was carried out on the data sets for a few plasma shaping coils to determine if fitting only two of the three field components would result in a significantly different solution for the position of the coil. As can be seen in Eq. 1a, any two of the three components is adequate to determine both the shift and the tilt of the coil. The test cases chosen represented poorer fits to one of the three components, typically $\mathrm{B}_{\phi}$. The results using any two components were found not to differ significantly $(<0.3$ standard deviation) from the fit using all three components. 


\section{RESULTS}

The results for the relative positions of the three coil systems and their anomalous field contributions are presented. In section 5.1 the positions of the plasma shaping coils, as measured at the four different apparatus locations, are transformed to a common axis and presented along with their average values (see section 3.3). The $\mathrm{M}_{0}$ apparatus position is (arbitrarily) chosen as the reference axis. In section 5.2, the location of the toroidal field axis is determined in the same reference frame. Section 5.3 treats the $n=1$ field of the ohmic coil. In section 5.4, the anomalous $n=1$ fields are transformed to the toroidal field axis to relate them to the present understanding of the fields experienced by the plasma. The relevant locations of the plasma chamber as determined from the mechanical location of the probe array and the saddle loop measurements of the chamber location are also presented. Section 5.5 presents results for the $n=1$ harmonic components for $m=0$ to 5 for the plasma shaping coils, and determines upper limits for the harmonic components of the ohmic heating and toroidal coil systems. Section 5.6 addresses the possibility of non-linear changes in the coil fields with coil current. Finally, section 5.7 discusses results for $\mathrm{n}=2$ and higher anomalies. In summary, it is found that the (small) anomalous fields are dominated by the contributions from the toroidal coil and the uncertainty is dominated by the uncertainty in the results for the toroidal coil.

\subsection{Plasma Shaping Coils}

The results for both $\boldsymbol{d}$ and $\boldsymbol{\alpha}$ for the plasma shaping coils are summarized in Figs. 5 and 6 , along with the final values obtained from a weighted average. The vectors denoting the shifts in the axes of data sets $\mathrm{M}_{65}, \mathrm{~L}$ and $\mathrm{U}$ related to $\mathrm{M}_{0}$ used in assembling the final data set are shown inset. Note that the uncertainties of the four measurements for each coil position often vary substantially. This results in the data appearing overly dispersive, where in fact the distribution is statistically acceptable. The positions of the coil centers fall into two groups, the innermost coils are closely clustered in a group contained in a circle about $3 \mathrm{~mm}$ in radius. The data shows a skew in the inner stack of coils of about $5 \mathrm{~mm}$ over its height. Most of the outer coils are clustered in a circle of about $5 \mathrm{~mm}$ in radius but displaced $6 \mathrm{~mm}$ in the $210^{\circ}$ direction from the inner coils. The values of $\boldsymbol{\alpha}$ are

randomly distributed, and since no coil is tilted by more than $4 \mathrm{~mm}$ measured at its edge, the values of $\boldsymbol{\alpha}$ may be considered proportionally smaller than the values of $\boldsymbol{d}$. 


\section{J.L. Luxon, et al.}

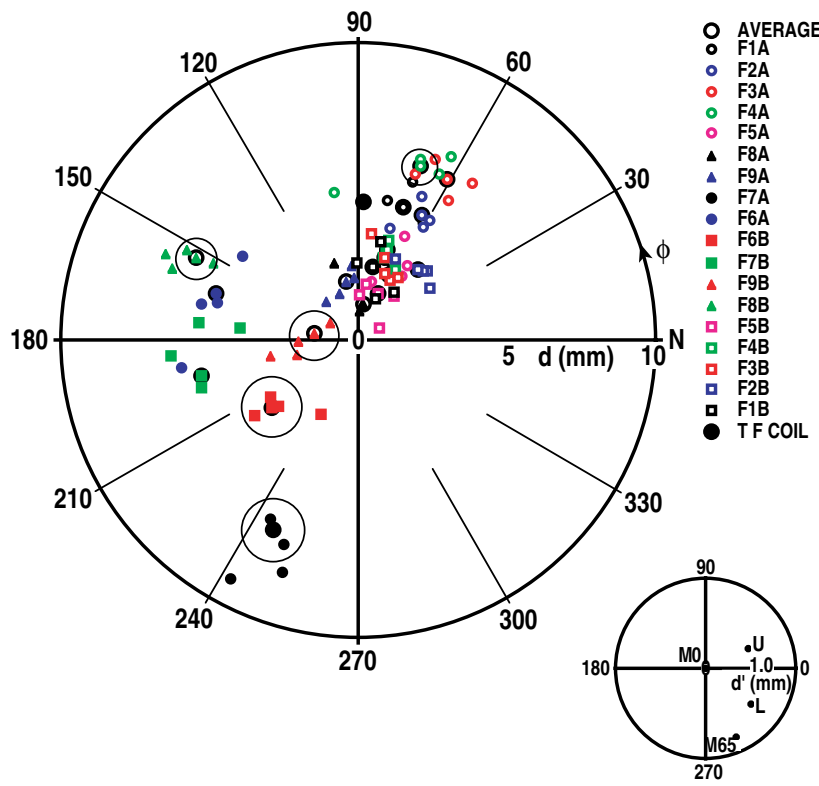

Fig. 5. The location of the plasma shaping coil centers $\boldsymbol{d}$ for each of the four data sets $\left(\mathrm{M}_{0}, \mathrm{~L}, \mathrm{U}\right.$, and $\left.\mathrm{M}_{65}\right)$ along with the weighted average of each (circled) and the toroidal field coil center all referenced to the $\mathrm{M}_{0}$ axes. The larger circles illustrate the uncertainty. Inset shows the location of the data set centers relative to $\mathbf{M}_{0}$.

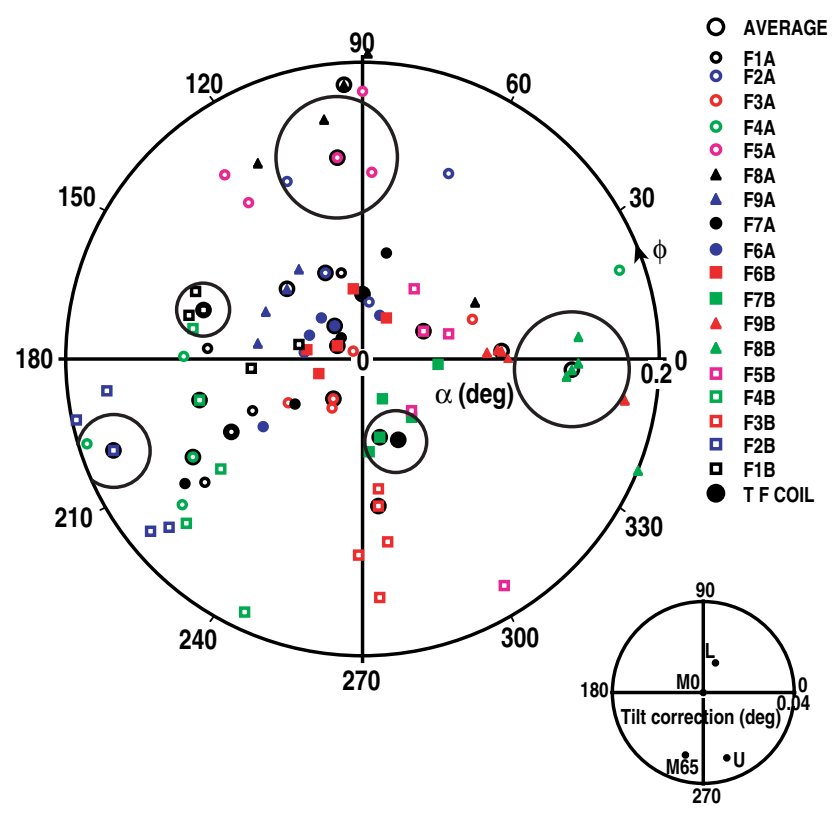

Fig. 6. The tilts $\boldsymbol{\alpha}$ of the plane of the plasma shaping coils for each of the four data sets $\left(\mathrm{M}_{0}, \mathrm{~L}, \mathrm{U}\right.$, and $\left.\mathrm{M}_{65}\right)$ along with the weighted average of each (circled) and the toroidal field coil all referenced to the $\mathrm{M}_{0}$ axes. The larger circles illustrate the uncertainty. Inset shows the tilt of the data set axes relative to $\mathbf{M}_{0}$. 


\subsection{Toroidal Field Coil}

Using the positions of the apparatus determined from the poloidal field coils, the horizontal positions of the toroidal field centers in the $\mathrm{M}_{0}$ frame are determined by solving for the locations $\mathrm{O}_{\phi}$ and $\mathrm{O}_{\mathrm{R}}$ where $\mathrm{B}_{1 \phi}=0$ or $\mathrm{B}_{1 \mathrm{R}}=0$, respectively (Fig. 7).

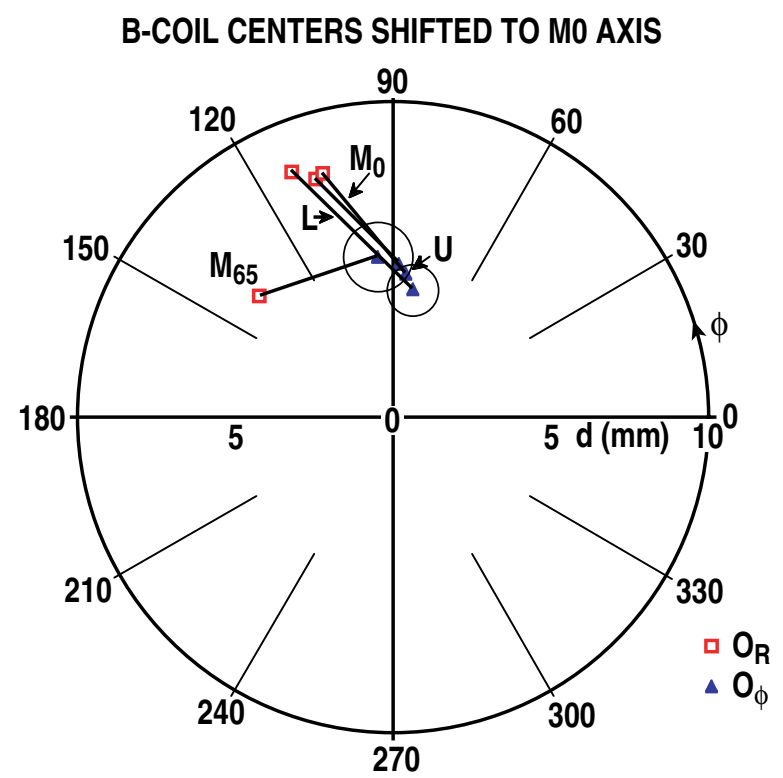

Fig. 7. Toroidal field coil centers shifted to the $\mathrm{M}_{0}$ axis for the final data sets. Corresponding centers $\mathrm{O}_{R}$ and $\mathrm{O}_{\phi}$ are connected with line segments. Circles illustrate the uncertainty.

The model for the toroidal coil accounted for the field due to the feed points of the toroidal coil; this field affects mostly the lower data set, since the feed-points end $50 \mathrm{~cm}$ below the midplane, and resulted in a shift of $0.4 \mathrm{~mm}$. For an ideal toroidal field, $\mathrm{O}_{\phi}$ and $\mathrm{O}_{\mathrm{R}}$ occur at a single point. In fact, $\mathrm{O}_{\phi}$ and $\mathrm{O}_{\mathrm{R}}$ are displaced from each other by $\Delta \cong 4 \mathrm{~mm}$, where $\Delta$ is the separation of the centers. From Eq. 1b, the displacements of $\mathrm{O}_{\mathrm{R}}$ axis from $\mathrm{O}_{\phi}$ axis could be indicative of a transverse error field given by $\mathrm{B}_{1 \mathrm{H}}=\Delta \cdot \mathrm{B}_{\mathrm{T}} /\left(2 \mathrm{R}_{\mathrm{m}}\right) \cong 25 \mathrm{G}$ (which would be indicative of $\mathrm{B}_{21} \cong 8 \mathrm{G}$ ) in the direction normal to the line between the centers (characteristic field values of the toroidal coil will always be referenced to $\mathrm{B}_{\mathrm{T} 0}=2.2 \mathrm{~T}$ ). The toroidal field center would then be at the midpoint of the two measurements. However, when the measurement apparatus was rotated $65^{\circ}, \mathrm{O}_{\mathrm{R}}$ rotated $65^{\circ}$ with respect to $\mathrm{O}_{\phi}$; this is indicative of a systematic error in the measurement apparatus. Since $\mathrm{O}_{\phi}$ did not shift within the measurement uncertainty, it represents the true axis of the toroidal field. Following correction of the position of the $\mathrm{O}_{R}$ in the $\mathrm{M}_{0}$ data set using the $\mathrm{M}_{65}$ data, $\mathrm{B}_{1 \mathrm{H}}$ is less than $8 \mathrm{G}(0 \pm 8 \mathrm{G})$. The $\mathrm{B}_{\mathrm{R}}$ probes are sensitive to first order to small misalignments about their vertical axes resulting in a contribution from the much larger $\mathrm{B}_{\phi}$. The shift seen here represents a systematic 
misalignment of $0.06^{\circ}$, which is several times the expected alignment errors and six times the rms variation seen in the data. No explanation for this error has been identified (the apparatus was disassembled and removed from the plasma chamber when the measurements were completed), and it is too small to affect poloidal coil measurements.

The transverse error field can also be evaluated from a second data set (Fig. 8). This data was taken as part of a preliminary run. The apparatus was disassembled, removed from the plasma chamber, realigned on the shop floor, disassembled and reinstalled between taking the preliminary and final data sets. Thus, the alignments for the two runs are independent. Here data were taken at three elevations and with the apparatus rotated by $24^{\circ}\left(\mathrm{M}_{24}\right)$ and $90^{\circ}\left(\mathrm{M}_{90}\right)$. This data set had the feature that several ferromagnetic objects were inadvertently in the plasma chamber between the elevations $\mathrm{z}=0.3 \mathrm{~m}$ and $0.8 \mathrm{~m}$ at $\mathrm{R} \cong 1.1 \mathrm{~m}$. Data from the poloidal coils and the toroidal coil were used to characterize the ferromagnetic objects, with the result that the ferromagnetic dipoles did not impact the toroidal field measurement. It is also expected that these objects would not mask the presence of a horizontal field. The measurements of the toroidal field centers at the lower level are particularly insensitive to the dipoles over $1 \mathrm{~m}$ above.

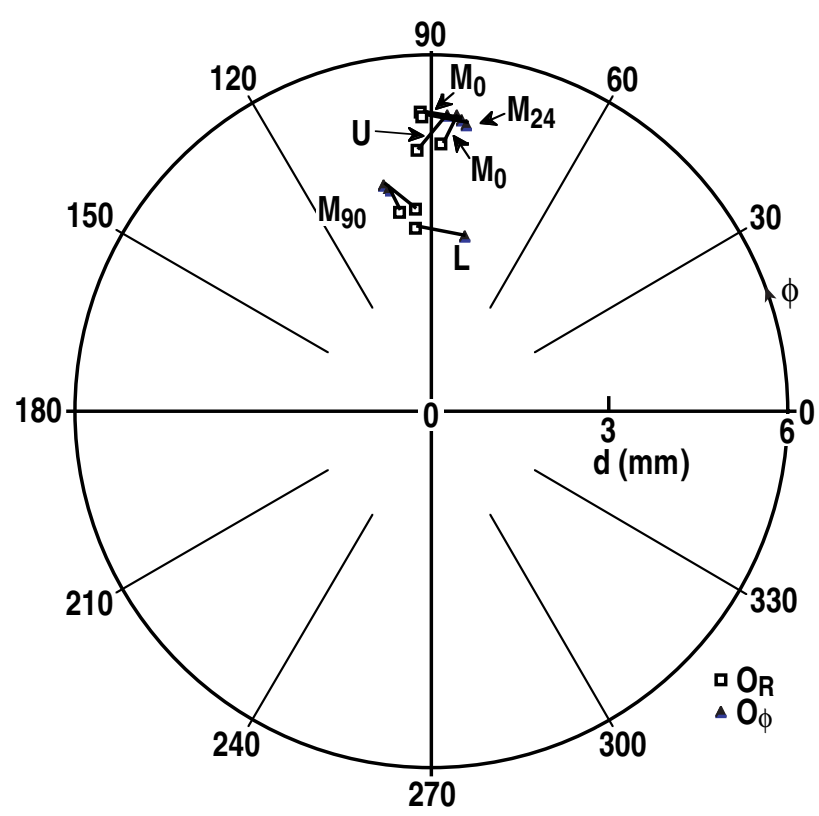

Fig. 8. Toroidal field centers for the preliminary data set without shifting to a common axis. Corresponding centers $\mathrm{O}_{\mathrm{R}}$ and $\mathrm{O}_{\phi}$ are connected with line segments (there are two almost identical $\mathbf{M}_{90}$ data sets).

Less accuracy was attained in the placement and alignment of the apparatus at each location for this data set, and the relative locations of the various data sets could not be adjusted to a common axis as was done for the final data set. However, the determination of the presence of a transverse field only depends on the separation between $O_{R}$ and $O_{\phi}$. 
ANOMALIES IN THE APPLIED MAGNETIC FIELDS ON

DIII-D AND THEIR IMPLICATIONS FOR THE

UNDERSTANDING OF STABILITY EXPERIMENTS

J.L. Luxon, et al.

These data (Fig. 8) are characterized by a separation between $\mathrm{O}_{\mathrm{R}}$ and $\mathrm{O}_{\phi}$ axes of less than $0.8 \pm 0.8 \mathrm{~mm}$ for each data set. Indeed, the rotation of the apparatus by $90^{\circ}\left(\mathrm{M}_{90}\right)$ done immediately following the $\mathrm{M}_{0}$ data appears to account for most of the possible horizontal field. The data clearly shows that $\mathrm{B}_{1 \mathrm{H}}$ is small, $\mathrm{B}_{1 \mathrm{H}}=0 \pm 7 \mathrm{G}$. Combining the results of these two data sets, the transverse error field is $B_{1 H}=0 \pm 6 \mathrm{G}$.

Measurements using the probe array cannot separate the value of the $\mathrm{z}$ component of a $n=1$ toroidal field coil error from an apparent $n=1$ field owing to a tilt of the coil. The plasma response to either is also the same, and the knowledge of the equivalent tilt of the toroidal field with respect to the poloidal field coils (along with the individual shifts and tilts of the poloidal coils) determines the net error fields that affect the plasma. The measured values of $\mathrm{B}_{1 \mathrm{Z}}$ (including a correction for the toroidal coil leads) are summarized in Fig. 9.

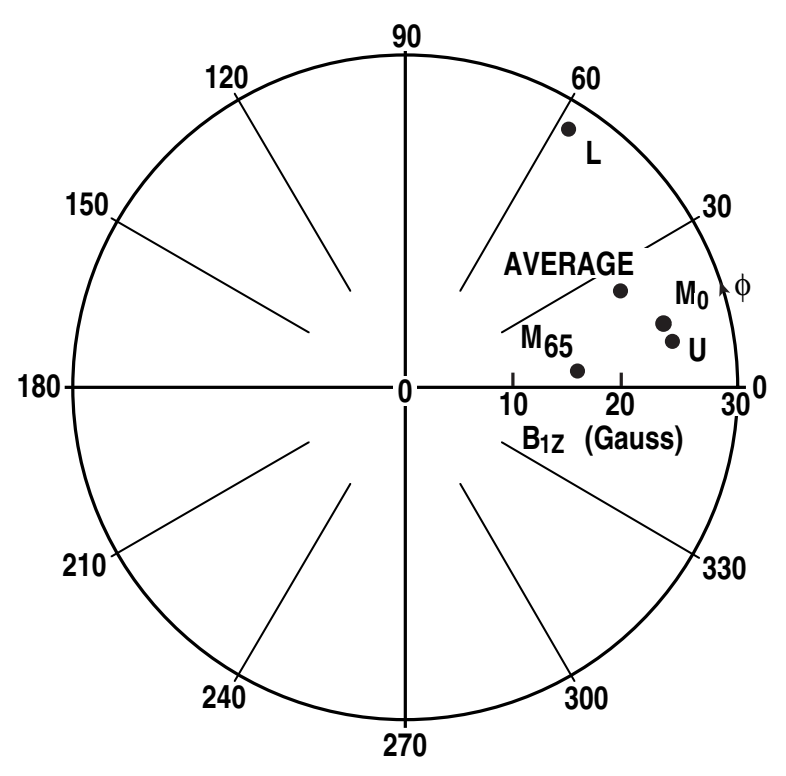

Fig. 9. Values of $B_{1 z}$ for the toroidal field coil from each data set. $B_{T 0}=-2.1 \mathrm{~T}$.

The average value of $\mathrm{B}_{1 \mathrm{Z}}$ (ave) $=21 \pm 8 \mathrm{G}$ is treated as the $\mathrm{B}$-coil plane being tilted by an angle $\alpha=0.06 \pm 0.02^{\circ}$ in the $290^{\circ}$ direction in the frame of apparatus at $\mathrm{M}_{0}$ (Fig. 6). This corresponds to a tilt of the toroidal field coil axis by $0.06^{\circ}$ from vertical in the $-250^{\circ}$ direction. Taking the average value as the best estimate of the tilt of the axis, the residual values of $B_{1 Z}$ are $<10 \mathrm{G}$.

Alternately, it can be assumed that the $\mathrm{U}$ and $\mathrm{L}$ measurements represent the values of $B_{1 Z}$ at these locations and use the average of the $M_{0}$ and $M_{65}$ values to represent $B_{1 Z}$ at the midplane. The result for $\mathrm{B}_{1 \mathrm{Z}}$ changes by only one half of the uncertainty. 


\subsection{Ohmic Heating Coil}

The ohmic heating coil is also a potential source of error fields. Although this coil is designed to produce small $n=0$ field components in the plasma region (such a design results in very little interaction between the plasma shaping and ohmic heating systems), turns displaced from the

intended coil axis can produce error fields. The observed $n=1$ fields produced by the ohmic coil at the midplane are as much as $3 \pm 0.5 \mathrm{G}$ for a full ohmic coil current of $137 \mathrm{kA}$ (which is reached at the very end of some discharges) and are dominated by a vertical component. This can be compared to the $n=1$ field from the most out of place coil (F7A) in a 1 MA discharge of $\sim 3 \mathrm{G}$. This field increases to $\mathrm{B}_{1} \cong 5 \mathrm{G}$ at $+0.75 \mathrm{~m}$ and $\mathrm{B}_{1} \cong 8 \mathrm{G}$ at $-0.75 \mathrm{~m}$, and both are predominantly vertical.

The ohmic heating coil was modeled by dividing it into seven sections, the solenoid, the upper and lower corner coils, the upper and lower end coils, and the upper and lower outer coils. There are not enough data to allow a fit of this many independent variables. Instead, the major components were placed in the positions indicated from the positions of the nearby coils that they are affixed to. The solenoid is wound on the toroidal field centerpost, and its axis was modeled in the location of the toroidal field axis. The outer turns are positioned adjacent to the field shaping coils they are encased with. Then, very small additional tilts of these coils were used to further optimize the fit to the data, and a good fit to the $n=1$ data was obtained. This result indicates that, although the positions of the ohmic heating coil turns cannot be modeled unambiguously, the measured fields are consistent with the turns being in positions consistent with the measured positions of the other coils.

\subsection{Anomalies Expressed in the Toroidal Field Frame of Reference}

The ultimate use of the multitude of data presented here is to determine the nature of the error fields associated with the tokamak and seen by the plasma. Thus, it is appropriate to transform all of the poloidal field data to the frame of the toroidal field coil. The reference axis is shifted in the horizontal plane $(\sim 5 \mathrm{~mm})$ and simultaneously tilted to the apparent axis of the toroidal field coil $\left(\sim 0.06^{\circ}\right)$ determined from $\mathrm{B}_{1 \mathrm{Z}}$ (ave) to best represent the error fields experienced by a confined plasma. This is summarized in Figs. 10 and 11 and in Table 2.

The magnitudes of the $n=1$ fields for the poloidal coils can then be calculated in this frame, and they are summarized in Figs. 12a and 12b. The magnitudes of the $\mathrm{n}=1$ fields normalized to a coil current of $2 \mathrm{kA}$ are $<2 \mathrm{G}$. Note that the value of $\Sigma \mathrm{B}_{1 \mathrm{R}}=2.06$ at $125^{\circ}$ for the plasma shaping coils includes the contribution due to tilting of the plasma shaping coil axis to the B-coil axis; without this (i.e., assuming the axis vertical), 
ANOMALIES IN THE APPLIED MAGNETIC FIELDS ON

DIII-D AND THEIR IMPLICATIONS FOR THE

UNDERSTANDING OF STABILITY EXPERIMENTS

J.L. Luxon, et al.

$\Sigma \mathrm{B}_{1 \mathrm{R}}=1.3 \mathrm{G}$ at $165^{\circ}$. The contribution of the global tilt to $\mathrm{B}_{1 \mathrm{Z}}$ is negligible. For a tokamak discharge, the coil currents are not all equal; the currents in the inner coils tend to be considerably lower than those in the outer coils. The $n=1$ fields are summarized in Figs. $12 \mathrm{c}$ and $12 \mathrm{~d}$ for discharge 65489 to allow comparison to the previous measurements ${ }^{3}$.

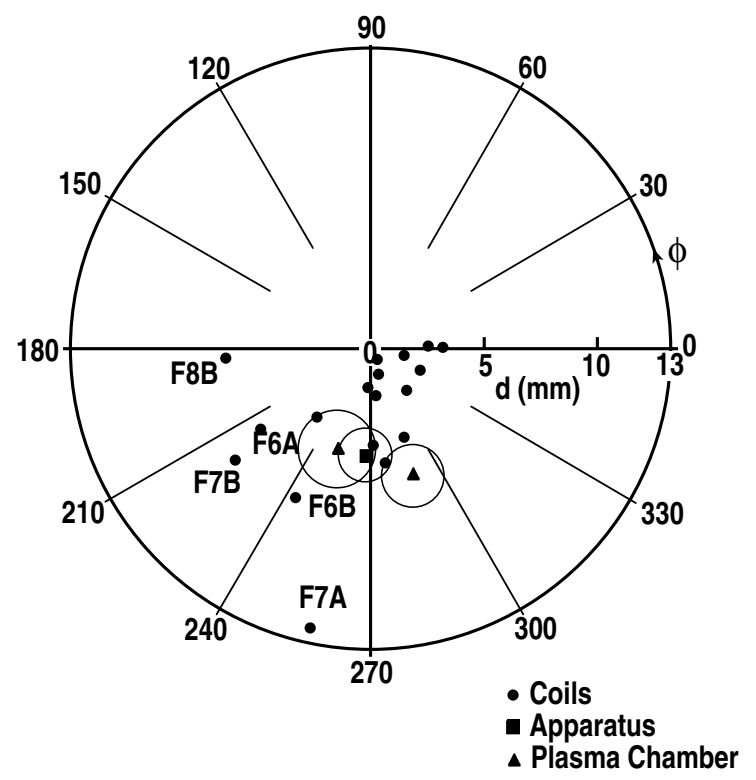

Fig. 10. The average values of $\boldsymbol{d}$ for the plasma shaping coils (Fig. 5) referenced to the toroidal field coil axes along with the locations of the plasma chamber and the measurement apparatus. Circles indicate the uncertainty.

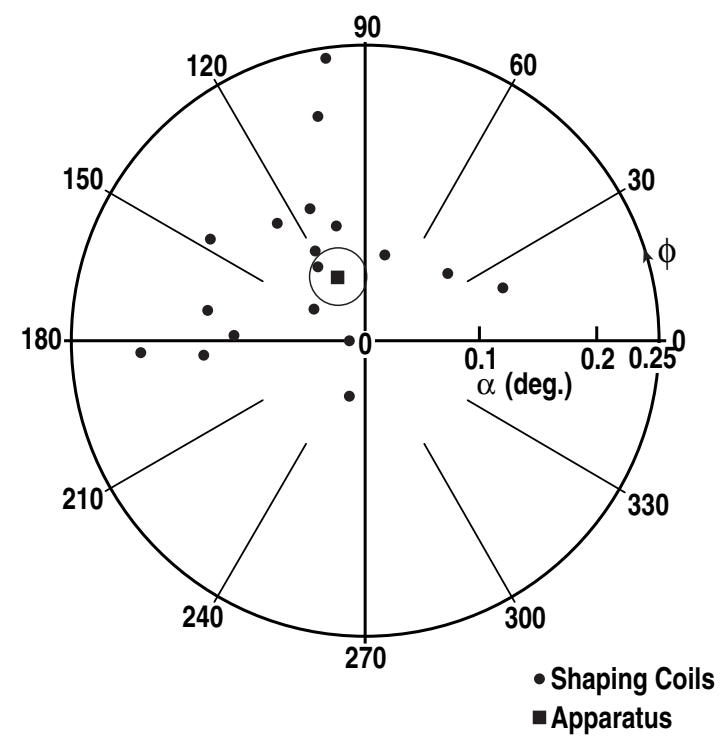

Fig. 11. The average values of $\boldsymbol{\alpha}$ for the plasma shaping coils (Fig. 6) referenced to the toroidal field coil axes. Circles indicate the uncertainty. 
ANOMALIES IN THE APPLIED MAGNETIC FIELDS ON

DIII-D AND THEIR IMPLICATIONS FOR THE

UNDERSTANDING OF STABILITY EXPERIMENTS

J.L. Luxon, et al.

B1R for Shaping Coil Currents $=-2 \mathrm{KA}$
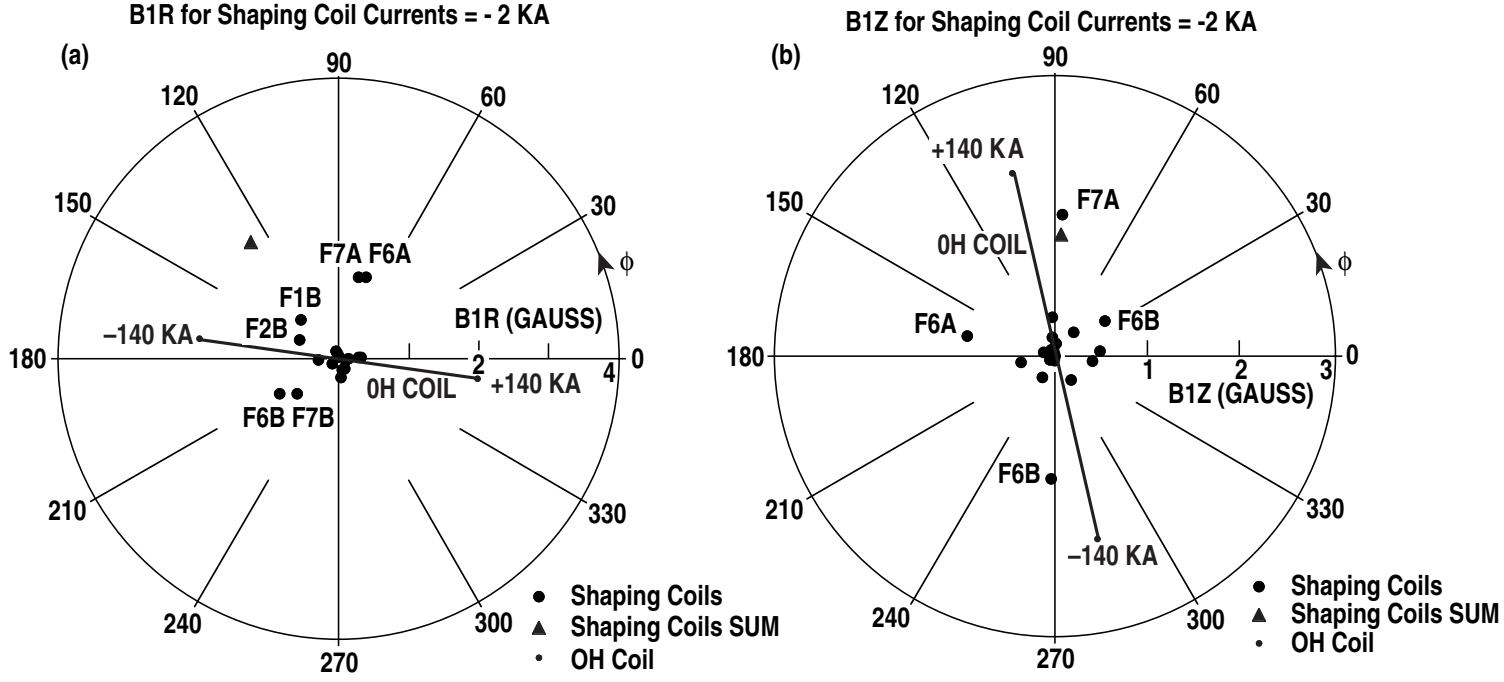

B1R for Discharge 65489

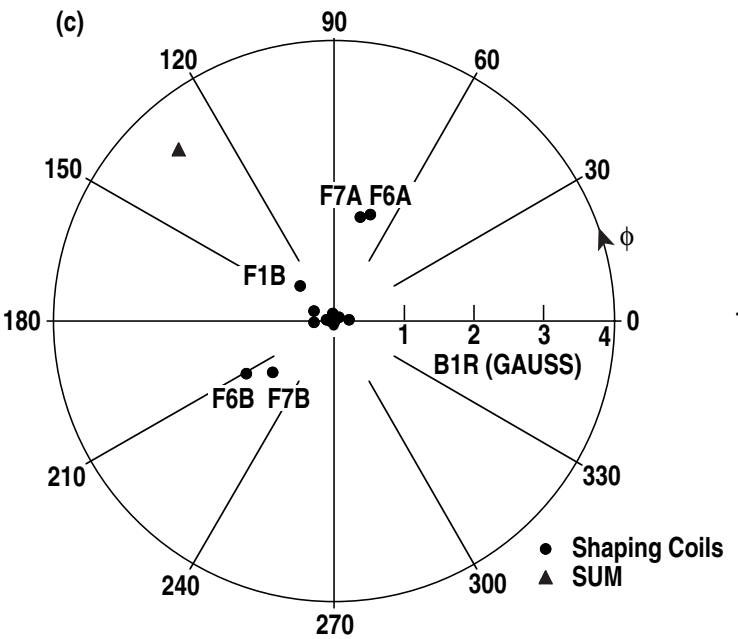

B1Z FOR DISCHARGE 65489

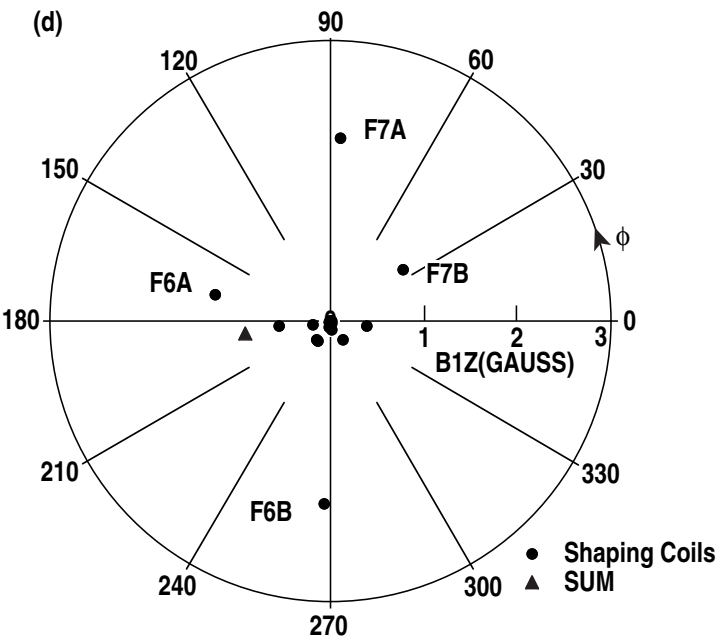

Fig. 12. The values of $\mathrm{B}_{1 \mathrm{R}}$ and $\mathrm{B}_{1 \mathrm{Z}}$ for the poloidal coils and the ohmic heating coil referenced to the axis of the toroidal field; a) and b) Coil currents at $2 \mathrm{kA}$. Superposed is a line indicating the ohmic heating coil field as a function of current. c) and d) For the coil currents of shot 65489 at $1900 \mathrm{~ms}$ (the contributions from the $\mathrm{OH}$ coil are negligible). 
J.L. Luxon, et al.
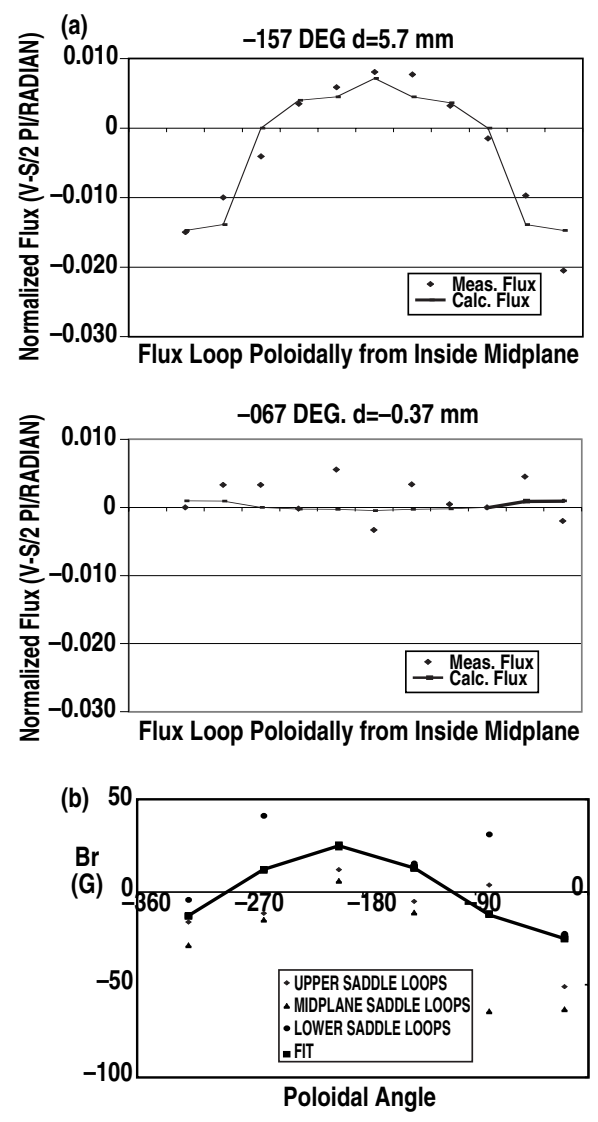

Fig. 13. Plasma chamber position in the toroidal field; a) from the two component $\left(-67^{\circ}\right.$ and $\left.-157^{\circ}\right)$ poloidal saddle loop array, b) from the toroidal saddle loop array.

From Fig. 10, it can be seen that these results are reasonably consistent with the center of the plasma chamber measured at the bottom of the central sleeve and used as the reference for the magnetic measurements. This is consistent with the original assembly measurements of the chamber that indicated rms deviations of $\pm 1 \mathrm{~mm}$ with concentricity of better than $\pm 1 \mathrm{~mm}$.

\subsection{Harmonic Field Analysis}

The field anomalies associated with coil systems are most useful to understanding the physics of the plasma when expressed in terms of the higher order field components $\mathrm{B}_{\mathrm{mn}}$ experienced by the plasma. These fields create islands in the magnetic structure where they resonate with rational flux surfaces in the plasma. For anomalies with known geometry, the values of $B_{m n}$ are calculated using Fourier harmonic analysis on the relevant flux surfaces. They depend on localized gradients in the error field, and are thus functions of the flux surface. Islands are made dominantly by the component of the error field normal to the flux surface. The results for the 2,1 fields on the $q=2$ surface of a typical plasma 
ANOMALIES IN THE APPLIED MAGNETIC FIELDS ON

DIII-D AND THEIR IMPLICATIONS FOR THE

UNDERSTANDING OF STABILITY EXPERIMENTS

J.L. Luxon, et al.

located on the measured toroidal field axis for the shaping coil positions given in Table 2 and with a coil current of $2 \mathrm{kA}$ are summarized in Fig. 1418.

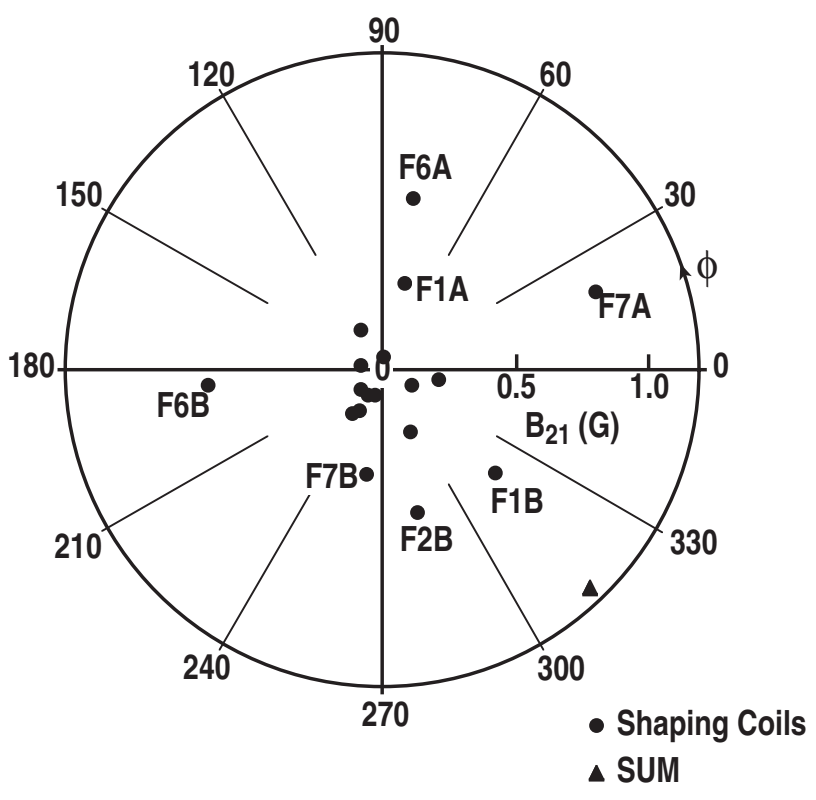

Fig. 14. The contributions to $B_{21}$ calculated on the $q=2$ surface of a typical elongated plasma from the plasma shaping coils referenced to the toroidal field axis for $\mathrm{I}=-2 \mathrm{kA}$.

These become a set of basis functions to be used with the coil currents to calculate the approximate 2,1 fields for any given discharge. The sum of the plasma shaping 2,1 fields for shot 65489 at $1900 \mathrm{~ms}$ is $\mathbf{B}_{21}{ }^{\mathrm{r}}(65489.1900)=0.63 \pm 0.25 \mathrm{G}$ at $55^{\circ}$, similarly for shot 103158 from Ref. $2, \mathbf{B}_{21}{ }^{\mathrm{r}}(103158.1300)=0.87 \pm .25 \mathrm{G}$ at $145^{\circ}$. It is appropriate to note that the small rotation of the reference axis from vertical to the toroidal field axis changed $\mathbf{B}_{21}{ }^{\mathrm{F}}$ substantially. The uncertainty is due mostly to the uncertainty in the tilt of the toroidal field.

The mode amplitudes for $\mathrm{m}=0-5, \mathrm{n}=1$ modes of the plasma shaping coils were calculated and are shown in Fig. 15a. As mentioned above, small changes in the tilt of the other coils with respect to the toroidal axis can change the amplitude of these modes significantly. The amplitude of the 1,1 mode is strongly dependent on the location in the horizontal plane of the toroidal field coil axis with respect to the axes of the shaping coils. If this location is moved to the approximate center of the outer plasma shaping coils, the amplitude of this mode decreases by an order of magnitude.

Upper limits on the 2,1 fields from the ohmic heating coil at full current are estimated by considering its component parts (Fig. 1) at full current. The contribution of the outer turns is estimated using the positions of the associated shaping coils with the result $\mathrm{B}_{21}{ }^{\mathrm{UH}}($ outer $)=0.1 \mathrm{G}$. The solenoid contributes $\mathrm{B}_{21}{ }^{\mathrm{UH}}$ (solenoid) $<0.5 \mathrm{G}$. The effect of 


\section{J.L. Luxon, et al.}

the seven top and seven bottom coils is estimated by considering a maximum displacement of $5 \mathrm{~mm}$ and scaling from the nearby shaping coil (F8A) with the result $\mathrm{B}_{21}{ }^{\mathrm{UH}}$ (ends) $<0.5 \mathrm{G}$. Thus the upper limit of the 2,1 field is $\mathrm{B}_{21}{ }^{\mathrm{UH}}<1.0 \mathrm{G}$ at $137 \mathrm{kA}$. Note that in many of the cases of interest, the current in the ohmic heating coil is $20 \mathrm{kA}$ or less and the related contribution to the 2,1 fields is negligible.

(a)

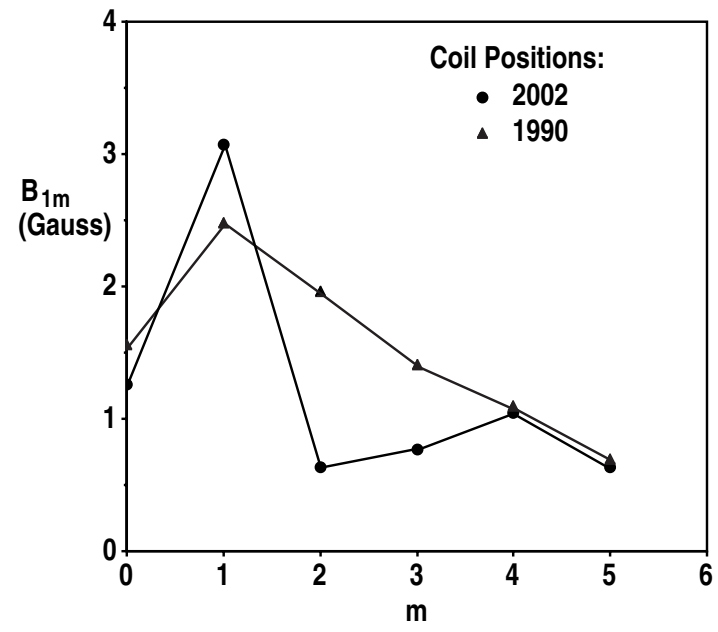

(b)

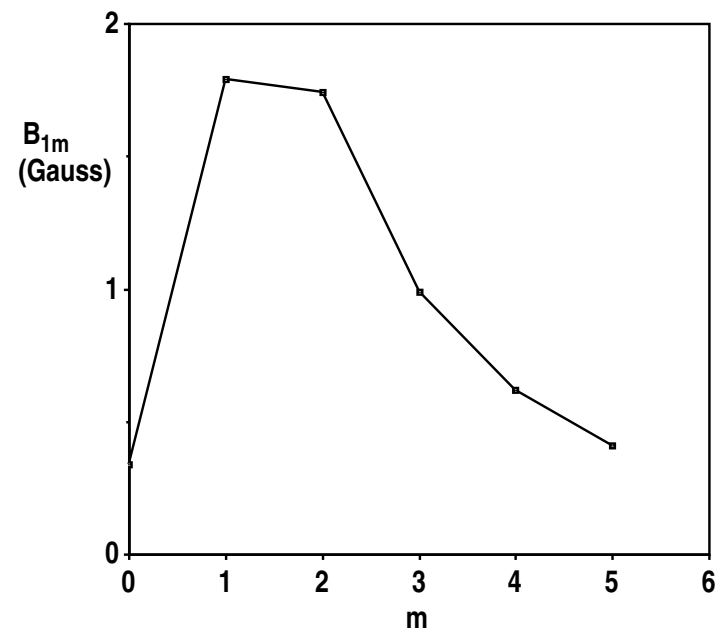

Fig. 15. a). The mode amplitudes of the $n=1$ modes with low $m$ for discharge 65489 at $1.900 \mathrm{sec}$. The values determined using the locations for the coils from $1990^{3}$ have been re-calculated on a typical elongated plasma $q=2$ surface. They differ somewhat $(10 \%$ for $\mathrm{m}=2)$ from the earlier calculations presumably owing to the use of a typical q=2 surface here to replace the earlier circular approximation. The 2001 values are calculated on the same plasma surface for the coil locations shown in Table 2. b) The mode amplitudes of the toroidal field coil buswork calculated on the same $\mathrm{q}=2$ surface. 
ANOMALIES IN THE APPLIED MAGNETIC FIELDS ON

DIII-D AND THEIR IMPLICATIONS FOR THE

UNDERSTANDING OF STABILITY EXPERIMENTS

J.L. Luxon, et al.

Toroidal field related $n=1$ error field components measured at $R_{m}$ are below our level to distinguish them $\left(\mathrm{B}_{1}{ }^{\mathrm{l}}<6 \mathrm{G}\right.$ at $\left.2.1 \mathrm{~T}\right)$ owing to the high background field present. Model calculations indicate that a reasonable upper limit on the 2,1 error fields from the toroidal field coil is one third of $\mathrm{B}_{1}{ }^{\mathrm{I}}$, or $\mathrm{B}_{21}{ }^{\mathrm{I}}<2 \mathrm{G}$. Error fields originating from the toroidal field coil must be due to either a) current not flowing where expected, b) the connecting bus-work, or c) turns out of position. Groups of six turns are bonded into 24 bundles, and it has been directly verified that the bundles carry the expected current. The known error field from the toroidal field buswork is calculated to contribute $\mathrm{B}_{21}$ (bus) $=1.7 \pm 0.4 \mathrm{G}$. The amplitudes of other $\mathrm{n}=1$ modes are similar to this (Fig. 15b). Assessments of the variation in the coil positions indicate that they are within a few $\mathrm{mm}$ of nominal. Statistical assessments of the field anomalies that would likely result from such coil variations have been made for other tokamaks ${ }^{19,26}$. These studies can be readily scaled to DIII-D and the results indicate that given a range of displacements of $\pm 3 \mathrm{~mm}$ the expected value of the field anomaly is small with $\mathrm{B}_{21}<0.5 \mathrm{G}$. Measurements made with local magnetic probes in a number of positions outside and in the bore of the tokamak (where the background field goes rapidly to zero) support the absence of any significant coil anomaly. This leads to the conclusion that the error field of the toroidal coil is likely dominated by the contributions from the buswork, $\mathrm{B}_{21}=1.7 \pm 0.4 \mathrm{G}$. Other $\mathrm{n}=1$ modes have similar or lesser amplitudes (Fig. 15b).

In summary, most of the plasmas of interest here and in other DIII-D plasma stability studies have $\mathrm{I}_{\mathrm{P}}<1.5 \mathrm{MA}$ and $\mathrm{I}_{\mathrm{OH}}<20 \mathrm{kA}$. Thus, the 2,1 fields are likely dominated by the contributions from the toroidal field coil, which sets an upper limit on them of $\mathrm{B}_{21}{ }^{1}<2 \mathrm{G}$ at $\mathrm{B}_{\mathrm{T}}=2.1 \mathrm{~T}$. Other low $\mathrm{m}, \mathrm{n}=1$ modes would have similar limits.

\subsection{Non-Linear Effects}

Non-linear changes in the fields with coil current are also a potential source of anomalous fields. There were no non-linearities in the $n=1$ field values observed for the individual coil systems. Toroidal field data was taken at both $0.35 \mathrm{~T}$ and $2.1 \mathrm{~T}$, ohmic heating coil at $20 \mathrm{kA}$ and $120 \mathrm{kA}$ and some of the outer poloidal coils at $2 \mathrm{kA}$ and $4 \mathrm{kA}$. For the different field strengths, the calculated position of the coil center did not change to within $\pm 0.1 \mathrm{~mm}$. Similarly, significant contributions from eddy currents were observed only rarely. They were less than $5 \%$ of the measured signals, appeared to have $n=2$ symmetry consistent with the locations of the neutral beamlines, and had a decay time of $1.5 \mathrm{~s}$, characteristic of eddy currents in the beamlines.

The possibility that the interaction of the poloidal and toroidal coil systems was producing a significant source of error was also investigated. Operation of a toroidal field coil in the fringing field of the poloidal coils (and plasma) exerts a twisting force on the toroidal coil turns. To lowest order this twisting produces a small vertical $(n=0)$ field that 
is inconsequential. Two mechanisms were used to determine the $n=1$ and higher contribution to the error fields from the interaction of the two coil systems. In the first, $\boldsymbol{d}$ and $\alpha$ were determined for the toroidal coil at $2.1 \mathrm{~T}$ with the ohmic heating coil at 0 and $100 \mathrm{kA}$. The change in $\boldsymbol{d}$ was less than $0.1 \mathrm{~mm}$ and the change in $\alpha$ less than $0.003^{\circ}$.

In the second method, the saddle loop arrays, which are positioned to reject the firstorder flux from the toroidal coil, were used as a sensitive detector for non-linear effects. Coils F7A, F6A, F6B, and F7B were operated at $5 \mathrm{kA}$ each, and the results were compared both with and without the toroidal field at $2.1 \mathrm{~T}$. This configuration has the advantages that the high currents in the outer shaping coils cause high transverse fields across the outer legs of the toroidal field coil and that the symmetry of the configuration causes most of the $n=0$ poloidal field to be parallel to the toroidal saddle loops, decreasing the background flux. The $n=0$ vertical twist-related fields averaged across the plasma chamber are less than $5 \mathrm{G}$. This in itself indicates that motion of the coils is likely not a major contributor to field errors. The corresponding $n=1$ error fields were found to be less than $2 \pm 2 \mathrm{G}$. Thus, interaction of the toroidal and poloidal coil systems produces no significant additional error field. This also rules out the possibility of interactions of the plasma and the toroidal field coil producing field errors.

\section{7. $\mathrm{n}=2$ and Higher Order Fields}

The $n=2$ component of the probe data can be used to determine the in-plane (elliptical) and out of plane (bending) distortion of the individual plasma shaping coils (e.g., Ref. 3). Analysis of this data showed that it was also subject to significant contribution from ferromagnetic objects, especially components of the neutral beamlines (located toroidally at $30^{\circ}, 150^{\circ}, 210^{\circ}$, and $330^{\circ}$ giving them $n=2$ but little $n=1$ symmetry with respect to the coil systems), and possibly to some $n=2$ error in the probe array. The results are summarized in Table 3 with the caveat that the results are likely not characteristic of coil distortion for those coils with weak $n=2$ fields. No significant $n=3$ contributions were noted, and combining the $\mathrm{M}_{0}$ and $\mathrm{M}_{65}$ data for a few outer coils sets, resulting in a synthesized 16 probe array for each field component, showed no evidence of significant modes in the range $3<n<7$. 
ANOMALIES IN THE APPLIED MAGNETIC FIELDS ON

DIII-D AND THEIR IMPLICATIONS FOR THE

UNDERSTANDING OF STABILITY EXPERIMENTS

J.L. Luxon, et al.

Table 3: Axisymmetric $n=2$ error fields for the plasma shaping coils from the midplane $\mathrm{M}_{0}$ probe array.

\begin{tabular}{|l|r|r|r|r|}
\hline \hline COIL & $\begin{array}{c}\text { B2R } \\
\text { AMP } \\
(\mathrm{G})\end{array}$ & $\begin{array}{c}\text { B2R } \\
\text { ANGLE } \\
\text { (degrees) }\end{array}$ & $\begin{array}{c}\text { B2Z } \\
\text { AMP } \\
(\mathrm{G})\end{array}$ & $\begin{array}{c}\text { B2Z } \\
\text { ANGLE } \\
\text { (degrees) }\end{array}$ \\
\hline F1A & 0.27 & 0 & 0.20 & 90 \\
F2A & 0.07 & 30 & 0.20 & 90 \\
F3A & 0.05 & 80 & 0.07 & 70 \\
F4A & 0.02 & 110 & 0.05 & 80 \\
F5A & 0.15 & 160 & 0.10 & 50 \\
F8A & 0.04 & 145 & 0.03 & 70 \\
F9A & 0.08 & 140 & 0.12 & 40 \\
F7A & 0.95 & 160 & 0.30 & 140 \\
F6A & 1.50 & 170 & 0.30 & 70 \\
F6B & 0.60 & 90 & 0.70 & 80 \\
F7B & 0.60 & 100 & 0.25 & 40 \\
F9B & 0.35 & 90 & 0.12 & 170 \\
F8B & 0.15 & 90 & 0.08 & 170 \\
F5B & 0.07 & 90 & 0.01 & 110 \\
F4B & 0.02 & 80 & 0.07 & 160 \\
F3B & 0.04 & 10 & 0.09 & 90 \\
F2B & 0.15 & 15 & 0.04 & 90 \\
F1B & 0.20 & 160 & 0.20 & 100 \\
\hline \hline
\end{tabular}




\section{DISCUSSION AND SUMMARY}

\subsection{Summary of Results}

New results have been obtained for the magnetic field anomalies and relative positions of the DIII-D coil systems, plasma chamber, and nearby ferromagnetic objects. Care was taken to eliminate a number of sources of systematic error and to provide redundant data. These results show that the individual poloidal field coil centers are generally concentric to within about $\pm 5 \mathrm{~mm}$ but that the outermost coils are displaced from the axis of the toroidal field coil by as much as $12 \mathrm{~mm}$. The axes of the coils are parallel to within $\pm 0.2^{\circ}$ and the axis of the toroidal field coil is tilted by $0.06 \pm 0.02^{\circ}$ with respect to vertical. The shaping coil positions (displacement and tilt) with respect to the toroidal field coil result in anomalous fields on a typical $q=2$ surface referenced to the toroidal coil axis of $\mathrm{B}_{21} / \mathrm{I}_{\mathrm{P}}<1 \mathrm{G} / \mathrm{MA}$, which are of marginal concern to DIII-D operation. An upper limit can be set on the contribution from the ohmic heating coil of $\mathrm{B}_{21}{ }^{\mathrm{UH}}(137 \mathrm{kA})<1 \mathrm{G}$. Note for the time of interest in most discharges $\mathrm{I}_{\mathrm{OH}}<20 \mathrm{kA}$ and this contribution is negligible. The sensitivity of the toroidal field measurements is limited by coupling to the strong background field. The toroidal field coil measurements put an upper limit on $n=1$ error $\mathrm{B}_{1}^{\mathrm{T}}(2.1 \mathrm{~T})$ of $6 \mathrm{G}(0 \pm 6 \mathrm{G})$. This sets an upper limit $\mathrm{B}_{21}{ }^{1}(2.1 \mathrm{~T})<2.0 \mathrm{G}$. It is likely that the error field is dominated by the (calculated) contributions from the buswork. Non-linear contributions to $\mathrm{B}_{1}$ (e.g., from the interaction of the toroidal and poloidal coil systems) were negligible. Thus, for most discharges, our knowledge of the 2,1 field contributions is limited by the uncertainty in our understanding of the contribution from the toroidal field coil.

These data indicate that the plasma shaping coil positions do not vary from nominal by as much as previously reported ${ }^{3}$, and the calculated $m, n=2,1$ field averaged over the plasma $q=2$ surface is less than half of the previously reported values. The measurements reported here have improved the accuracy of the individual plasma shaping coil measurements by making measurements at multiple level in the plasma chamber and have removed important sources of systematic error especially owing to ferromagnetic structures. The results for the tilts of the coil planes are within expectations of the original installation and the results for the shifts from a common axis are about twice the expectation $^{11}$. Both this and the earlier measurement are referenced to the axis of the toroidal field, albeit by different techniques. However, the value reported here for the tilt of the axis of the toroidal field differs significantly from the earlier measurement. Here we 
ANOMALIES IN THE APPLIED MAGNETIC FIELDS ON

DIII-D AND THEIR IMPLICATIONS FOR THE

UNDERSTANDING OF STABILITY EXPERIMENTS

J.L. Luxon, et al.

\subsection{Relationship to DIII-D Plasma Experiments}

The measurements reported here do not support the presence of a substantial error field in the DIII-D plasma chamber associated with either the coils, especially the toroidal field coil, or a ferromagnetic object as suggested in Refs. 1 and 2. The strong toroidal field dependent term hypothesized in Ref. 1 was not found. Comparison to this experiment is made difficult by the fact that Ref. 1 uses shaping coil positions from the 1990 measurements ${ }^{3}$. Nevertheless, this issue should not substantially change their identification of a toroidal field related anomalous field of $\mathrm{B}_{21} / \mathrm{B}_{\mathrm{T}} \sim 3.4 \mathrm{G} / \mathrm{T}$. The upper limit of the directly measured toroidal field coil related 2,1 field found here is $\mathrm{B}_{21} / \mathrm{B}_{\mathrm{T}} \sim 1 \mathrm{G} / \mathrm{T}$. This suggests that while the locked-mode experiments found an optimal magnetic field configuration for operating the tokamak, this optimum does not correspond to a minimum in the external 2,1 error field. In addition, the scaling relationship found in Refs. 1 and 7 for the locked mode density $\mathrm{n}_{\mathrm{LM}}$ might have to be reexamined.

Resistive wall mode experiments identified a similar unknown field component ${ }^{2}$. In these experiments (shot 103158 at $1300 \mathrm{~ms}$ with $\mathrm{I}_{\mathrm{p}}=1.25 \mathrm{MA}$ and $\mathrm{B}_{\mathrm{T} 0}=-2.1 \mathrm{~T}$ ), a field $\mathrm{B}_{21}=7 \mathrm{G}$, which we calculate to be at an angle of $125^{\circ}$ using the DIII-D data base, was applied to optimally stabilize the RWM. The 2,1 fields calculated for this discharge using the shaping coil positions given in Table 2 are $B_{21} F=0.87 \pm 0.2 \mathrm{G}$ at $140^{\circ}$, $\mathrm{B}_{21}{ }^{\mathrm{OH}}(11 \mathrm{kA}) \sim 0$, and $\mathrm{B}_{21}{ }^{\mathrm{T}}<2 \mathrm{G}$. Thus, there is an unaccounted field of at least $4 \mathrm{G}$ $(6.1 \pm 2 \mathrm{G})$. It is perhaps noteworthy that the experimentally applied correction field ${ }^{2}$ and the calculated field determined here for the shaping coils are in essentially the same direction, but they differ in amplitude by nearly an order of magnitude. However, if the correction field was canceling an intrinsic field including resonant amplification ${ }^{17}$, the applied field and intrinsic fields would be $180^{\circ}$ apart.

There are similarities in the nature of the "missing" field reported in the locked mode and RWM experiments. Both experiments are carried out in diverted discharges. The results have been reproducible over a considerable period of time. The RWM report notes that the angular location of the field from the $\mathrm{C}$-coil that optimized performance is the same as the location (within $15^{\circ}$ ) for the field correction postulated based on the earlier locked mode experiments, suggesting that the apparent anomalous field was essentially in the same location. The RWM experiments identified the field component as 2,1, but there was no unique characteristic feature (e.g., from magnetic diagnostics) identified. There was no $\mathrm{q}=1$ surface in the plasma, apparently excluding the possibility of a 1,1 mode. The locked mode experiments performed a least square minimization to determine the contributions from 1,$1 ; 2,1$; and 3,1 modes with the result that the 2,1 mode was dominant. However, it appears that the regression analysis used may not produce a unique solution, and there was no unique signature identified to characterize the mode. Thus, both experiments 
characterize the missing field as being at the same location and of comparable magnitude, but the identification of the mode in both cases is circumstantial.

The experimental conditions for the locked mode and RWM results are quite different. The locked mode experiments are conducted in low density ohmically heated plasmas over a range of toroidal field. The RWM experiments are conducted at one toroidal field value in neutral beam heated moderate density plasmas with the normalized $\beta$ above the resistive wall limit. Thus we see that the phenomenon of an apparent missing field component is present (in diverted discharges) over a fairly wide range of experimental conditions.

These conflicting results between the stability experiments and the measured fields challenge our understanding of the interaction of the plasma with the confining field. We have ruled out hardware-related sources of error fields as explanations of the high 2,1 fields $\mathrm{B}_{21} \sim 5 \mathrm{G}$ needed to optimally stabilize $1 \mathrm{MA}, 2.1 \mathrm{~T}$ DIII-D tokamak discharges against locked modes and RWMs. 2,1 components of the shaping coil fields are dependent on detailed understanding of the coil positions, but are limited to $<\sim 1$ G/MA. The toroidal coil 2,1 field is less than $2 \mathrm{G}$ at full field (2.1T). The 1,1 mode for the plasma shaping coils is considerably larger than the 2,1 mode, but in the case of the locked-mode experiments, the coefficient for viscous coupling is expected to be a fraction of unity ${ }^{4}$, and the mode appears of diminished importance. For the resistive wall mode experiments, it was reported that there was no $\mathrm{q}=1$ surface in the plasma. Other modes, thought to be generally less significant to the discharge, are of lesser amplitude.

This conundrum raises the possibility that either local optimums in plasma performance have been encountered that do not represent a minimum in the 2,1 field, or that the models for the interaction of the plasma with the external field are inadequate. The former is at least plausible since the starting points of the stability experiments are usually with the external error correction systems operating at fields determined using the earlier coil positions of Ref. 3, but the existence of a local minimum still is not understood. In either case these results raise serious concerns about the present physics models for understanding the interaction of the plasma with anomalies in the confining field. One possibility is that the error field correction system used on DIII-D introduces unforeseen complications. This correction system uses a picture frame coil set that produces a dominantly radial field component at the midplane, which is, based on harmonic analysis, adjusted to cancel the 2,1 fields from other sources. This correction field is very different in structure from the field of the coil-related anomalies. It is also possible that an unrecognized non-axisymmetric current is flowing in the region of the plasma chamber from the separatrix outward. If part of the current path were in the chamber wall, a considerable energy source would be needed. It has been pointed out that the assumption of toroidal axisymmetry, needed to get from the force balance equations to the 
ANOMALIES IN THE APPLIED MAGNETIC FIELDS ON

DIII-D AND THEIR IMPLICATIONS FOR THE

UNDERSTANDING OF STABILITY EXPERIMENTS

J.L. Luxon, et al.

Grad-Shafranov equation, is a demanding one ${ }^{21}$. It is assumed that islands in the plasma and small perturbations in the external field can be treated as perturbations. Perhaps this formulation is incorrect. Possibly the small net shift or tilt of the poloidal coils from the axis of the toroidal field is not treated properly by these models or the plasma is not in fact aligned with the toroidal field. Boozer has suggested models for the resonant amplification of small error fields ${ }^{17}$, but this is inconsistent with the observation in the previous paragraph that the additional field applied to optimize the plasma is in the same direction as the calculated 2,1 field for the shaping coils.

These results also show the inadequacy of using the data from plasma measurements coupled with our present understanding of the physics of the plasma's interaction with the magnetic field to deduce the coil related error fields $1,15,16$ in the absence of direct measurements. Finally, without better understanding of these issues, it appears difficult to project the required limits on hardware related error fields to the design of next generation tokamaks 7 .

Measurements are being undertaken on the DIII-D tokamak to better understand the plasma response to applied non-axisymmetric fields with the intention of clarifying or resolving the issues raised here. The experiments should benefit considerably from the addition of the I-coil, an array of 12 picture frame coils mounted on the inside plasma chamber wall above and below the midplane. These coils, along with the existing six coil midplane array, result in an array of coils three high and six in circumference located symmetrically about the outer midplane of the tokamak. This array will allow a wider range of more narrowly defined mode spectra to be applied to the plasma, and will hopefully allow the plasma response to the various modes to be better elucidated. 


\section{REFERENCES}

[1] Scoville, J.T., R.J. La Haye, Nucl. Fusion 43 (2003) 250

[2] Garofalo, A.M., R.J. La Haye, and J.T. Scoville, Nucl. Fusion 42 (2002) 1335

[3] La Haye, R.J., J.T. Scoville, Rev. Sci. Instrum. 61 (1991) 2146

[4] Fitzpatrick, R., Nucl. Fusion 33 (1993) 1049

[5] Wesson, J., Tokamaks, Clarendon Press, Oxford (1997)

[6] Buttery, R.J., et al., Nucl. Fusion 39 (1999) 1827

[7] ITER Physics Basis Editors, Nucl. Fusion 39 (1999) 2251

[8] Meade, D.M., et al., Fusion Energy 2002 (Proc. 19th Int. Conf. Lyon, 2002)

(Vienna:IAEA)CD-ROM file FT2-6 and

http://www.iaea.org/programmes/ripc/physics/fec2002/pdf/ft2_6.pdf

[9] La Haye, R.J., “The Physics of Locked Modes in ITER, Error Field Limits, Rotation for Obviation and Measurement of Error Field" General Atomics report GA-A22468 (1997)

[10] Buttery, R.J., et al., Nucl. Fusion 40 (2000) 807

[11] Luxon, J.L., Nuclear Fusion 42 (2002) 614

[12] Strait, E.J., et al., Phys. Plasmas 4 (1997) 1783

[13] Turnbull, A.D., et al., Nucl. Fusion 42 (2002) 917

[14] Hender, T.C. et al., Nucl. Fusion 32 (1992) 2091

[15] Fishpool, G.M., P.S. Haynes, Nucl. Fusion 34 (1994) 109

[16] Jackson, G.L., et al., Rev. Sci. Instrum. 60 (1989) 2883

[17] Boozer, A.H., Phys. Rev. Lett. 86 (2001) 1176 


\section{ACKNOWLEDGMENTS}

This work is supported by the U.S. Department of Energy under Contract Nos. DE-AC03-99-ER54463 and DE-AC02-76-CH03073. We thank R.J. La Haye for access to the 1990 data and detailed discussions of the earlier DIII-D field error measurements. We would also like to thank J. Hovland and E. Taylor for their technical support. 